\title{
Association Control based \\ Load Balancing in Wireless \\ Cellular Networks using \\ Preamble Sequences
}

by

Ankit Chopra

\author{
A thesis \\ submitted to the Victoria University of Wellington \\ in fulfilment of the \\ requirements for the degree of \\ Master of Engineering \\ in Network Engineering.
}

Victoria University of Wellington

2012 



\begin{abstract}
The efficient allocation and use of radio resources is crucial for achieving the maximum possible throughput and capacity in wireless networks. The conventional strongest signal-based user association in cellular networks generally considers only the strength of the signal while selecting a BS, and ignores the level of congestion or load at it. As a consequence, some BSs tend to suffer from heavy load, while their adjacent BSs may carry only light load. This load imbalance severely hampers the network from fully utilizing the network capacity and providing fair services to users.

In this thesis, we investigate the applicability of the preamble code sequence, which is mainly used for cell identification, as an implicit information indicator for load balancing in cellular networks. By exploiting the high auto-correlation and low cross-correlation property among preamble sequences, we propose distributed load balancing schemes that implicitly obtain information about the load status of BSs, for intelligent association control. This enables the new users to be attached to BSs with relatively low load in the long term, alleviating the problem of non-uniform user distribution and load imbalance across the network.

Extensive simulations are performed with various user densities considering throughput fair and resource fair, as the resource allocation policies in each cell. It is observed that significant improvement in minimum throughput and fair user distribution is achieved by employing our proposed schemes, and preamble sequences can be effectively used as a leverage for better cell-site selection from the viewpoint of fairness provisioning. The load of the entire system is also observed to be balanced, which consequently enhances the capacity of the network, as evidenced by the simulation results.
\end{abstract}




\section{Acknowledgments}

I would like to express my deepest gratitude to my supervisor Dr. Winston K.G. Seah for his unrelenting motivation and criticism, without which, this work would not have been possible. I also thank Peter Sam Raj for his help with simulations and very thoughtful suggestions. 


\section{Contents}

1 Introduction 1

1.1 Cellular Wireless Networks . . . . . . . . . . . . . . . 1

1.1.1 Challenges in Cellular Networks . . . . . . . . . 5

1.1.2 Issue of Non-Uniform User Distribution . . . . . . . . 6

1.2 Thesis Objective . . . . . . . . . . . . . 7

1.3 Thesis Contributions . . . . . . . . . . . . 8

1.4 Thesis Organization . . . . . . . . . . . . . . . . 9

2 Related Work 11

2.1 Load Balancing in Cellular Networks . . . . . . . . . . . . . . 11

2.1.1 Channel Assignement Schemes . . . . . . . . . . . . . 11

2.1.2 Dynamic Load Balancing Schemes . . . . . . . . . . 12

2.1.3 Schemes based on Association Control . . . . . . . . 14

2.1.4 Relay Assisted Traffic Transfer Schemes . . . . . . . . 15

2.2 Load Balancing in Wireless Local Area Networks . . . . . . . 17

2.3 Recent Research Work . . . . . . . . . . . . . . . . 18

2.4 Summary . . . . . . . . . . . . . . . . . . 19

3 System Model $\quad 21$

3.1 Definitions ....................... 21

3.1.1 Preamble Sequences . . . . . . . . . . . . . 21

3.1.2 Fairness in Wireless Networks . . . . . . . . . . . 23

3.2 Utilizing Preamble Sequences for Load Balancing . . . . . . 25 
3.2.1 Load Balancing parameter . . . . . . . . . . . . . . . . . . . . . . . . . .

3.3 System Model . . . . . . . . . . . . . . . . . . . 28

3.4 Summary ....................... 30

4 Auto-Correlation based Implicit Load Indication Scheme 31

4.1 The Auto-Correlation Property . . . . . . . . . . . . . . 31

4.2 Preamble Modification Algorithm . . . . . . . . . . . 34

4.3 MS Cell Decision using AutoCorrelation . . . . . . . . . . 35

4.4 Summary . . . . . . . . . . . . . . . . . 37

5 Cross-Correlation based Implicit Load Indication Scheme 39

5.1 The Cross-Correlation Property . . . . . . . . . . . . . . 39

5.2 Preamble Allocation using Cross-correlation . . . . . . . . . 41

5.3 BS Selection Algorithm using Implicit Indication . . . . . . . 42

5.4 Summary . . . . . . . . . . . . . . . . . . . 44

6 Simulation Results and Discussion 45

6.1 Simulation Setup . . . . . . . . . . . . . . . 45

6.1.1 Channel Characteristics . . . . . . . . . . 46

6.1 .2 Simulation Environment . . . . . . . . . . . . 46

6.2 Simulation Results and Discussion . . . . . . . . . . . . 47

6.2.1 Load Variance Test . . . . . . . . . . . . . . . . . . . 49

6.2.2 Resource Fairness Test . . . . . . . . . . . . 51

6.2.3 Spectral Efficiency Test . . . . . . . . . . . . 52

6.2.4 Evaluation using Jain's Fairness Index . . . . . . . . . 55

6.3 Performance under Different Environments . . . . . . . . . . 57

6.3.1 Hata Model for a Small City . . . . . . . . . . . . . 58

6.3.2 Hata Model for a Large City . . . . . . . . . . . . . . 61

6.4 Comparsion against RSSI-based Schemes . . . . . . . . . . 65

6.5 Summary ....................... 67

$\begin{array}{llr}7 & \text { Conclusion } & 69\end{array}$ 
$\begin{array}{lll}8 & \text { Future Work } & 71\end{array}$ 


\section{List of Figures}

1.1 System architecture of a conventional cellular system _... 2

1.2 Frequency reuse . . . . . . . . . . . . . . 3

2.1 Channel borrowing without locking (CBWL). . . . . . . . 13

2.2 Cell breathing (decrease in coverage area with increase in subscribers $\ldots \ldots \ldots \ldots \ldots \ldots$

3.1 A sample pseudo-noise sequence . . . . . . . . . . . . . . . . 21

3.2 M-Sequence generator . . . . . . . . . . . . . . . 23

3.3 At central controller the metric is the number of peaks in the auto-correlation plot. . . . . . . . . . . . . . . 26

3.4 At central controller the metric is the value of cross-correlation with particular sequence. . . . . . . . . . . . . . . 26

3.5 A two cell model showing the concept of " $r$ ". . . . . . . . 27

3.6 Architecture of the Korean WiMax Broadband [30] . . . . . 28

4.1 Auto-correlation function of the sinewave . . . . . . . . . 32

4.2 Auto-correlation plot of a preamble of length 284 bits made with 4 repeated sequences of length $71 . \ldots \ldots$. . . . . 33

5.1 Two time series $\mathrm{x}, \mathrm{y} . \ldots \ldots \ldots \ldots$

5.2 The cross-correlation series with a maximum delay of 4000 showing a strong correlation at a delay of about $40 . \ldots 41$

6.1 A conventional 12-cell model with focus on cells 0,1 and 2 . 47 
6.2 Distribution of users in cells at $r=10 \ldots \ldots$. . . . . . 48

6.3 Load Variance test with 100 users in cell 0,80 users in cell 1, and 20 users in cell 3. . . . . . . . . . . . . . . 50

6.4 Load Variance test with 60 users in cell 0, 40 users in cell 1, and 10 users in cell $2 . \ldots \ldots$. . . . . . . . . . 50

6.5 Resource Fairness with initially 100 users in cell 0, 80 in cell 1 and 20 users in cell 2 . . . . . . . . . . . . . . . 51

6.6 Resource Fairness with initially 50 users in cell 0, 40 in cell 1 and 10 users in cell 2 . . . . . . . . . . . . . . . . 52

6.7 Throughput Fairness with initially 100 users in cell 0, 80 in cell 1 and 20 users in cell 2. . . . . . . . . . . . . . . 53

6.8 Throughput Fairness with initially 50 users in cell 0, 40 in cell 1 and 10 users in cell 2. . . . . . . . . . . . . . . . . . . 54

6.9 Load Balancing Index vs $r \ldots \ldots$. . . . . . . . . . 56

6.10 Jain's Fairness Index vs $r \ldots \ldots$. . . . . . . . . . 56

6.11 Throughout Fair vs $r$ for a small city . . . . . . . . . . . 59

6.12 Resource Fair vs $r$ for a small city . . . . . . . . . . . . . . 59

6.13 Load Variance vs $r$ for a small city . . . . . . . . . . . . 60

6.14 Load Balance Index vs $r$ for a small city . . . . . . . . . 61

6.15 Throughout Fair vs $r$ for a large city . . . . . . . . . . 62

6.16 Resource Fair vs $r$ for a large city . . . . . . . . . . . 63

6.17 Load Variance vs $r$ for a large city . . . . . . . . . . . . . 64

6.18 Load Balance Index vs $r$ for a large city . . . . . . . . . 65

6.19 Minimum Throughput gain vs $r \ldots \ldots 66$

6.20 Jain's Fairness Index vs $r \ldots \ldots 66$ 


\section{List of Tables}

4.1 Preamble Modification Algorithm (PMA) . . . . . . . . . . 35

4.2 MSCD Algorithm . . . . . . . . . . . . . . 36

6.1 Channel parameters for simulation . . . . . . . . . . . 48

6.2 Standard coverage parameters for the Hata model . . . . . . 57 


\section{List of Abbreviations}

$\begin{array}{ll}\text { AP } & \text { Access Point } \\ \text { AMC } & \text { Adaptive Modulation and Coding } \\ \text { ASN } & \text { Access Network } \\ \text { Autocorr } & \text { Autocorrelation } \\ \text { BS } & \text { Base Station } \\ \text { CAZAC } & \text { Constant Amplitude Zero Autocorrelation Waveform } \\ \text { CDMA } & \text { Code Division Multiple Access } \\ \text { CBWL } & \text { Channel Borrowing Without Locking } \\ \text { CBWLCR } & \text { CBWL with Channel Rearragement } \\ \text { CBWLnR } & \text { CBWL without Channel Arrangement } \\ \text { CSN } & \text { Connectivity Service Network } \\ \text { DCA } & \text { Dynamic Channel Assignment } \\ \text { FCA } & \text { Fixed Channel Assignment } \\ \text { FDMA } & \text { Frequency Division Multiple Access } \\ \text { FFT } & \text { Fast Fourier Transform } \\ \text { HCA } & \text { Hybrid Channel Assignment } \\ \text { LBSB } & \text { Load Balancing with Selective Borrowing } \\ \text { LFSR } & \text { Linear Feedback Shift Register } \\ \text { MACA } & \text { Mobile Assisted Call Admission } \\ \text { MCN } & \text { Multihop Cellular Networks } \\ \text { MLS } & \text { Maximum Length Sequences } \\ \text { MS } & \text { Mobile Station } \\ \text { MSCD } & \text { MS Cell Decision }\end{array}$


MTSO Mobile Telephone Switching Office

PAC Preamble Allocation using Cross-correlation

PMA Preamble Modification Algorithm

PN Pseudo Noise

PSTN Public Switched Telephone Network

QOS Quality of Service

RAS Radio Access Stations

RF Resource Fair

RSSI Received Signal Strength Indicator

SNR Signal to Noise Ratio

TF Throughput Fair

UCAN Unified Cellular and Ad hoc Network

WiBro Wireless Broadband

WLANSs Wireless Local Area Networks

WiMAX Worldwide Interoperability for Microwave Access

WSM WiMAX System Manager 


\section{Chapter 1}

\section{Introduction}

The popularity of wireless communication has made great gains over the past two decades as witnessed by the widespread adoption of Wireless Local Area Networks (WLANSs), cellular networks, and worldwide interoperability for microwave access (WiMAX) [3]. Users of these wireless access networks expect the highest quality, reliability, and ease of access to high-speed services. In terms of mobile communications, cellular networks have been a dominant player in the recent years and stands as the focus of our research in this thesis.

\subsection{Cellular Wireless Networks}

A cellular network provides mobile stations (MSs) or cell phones, with wireless access to the public switched telephone network (PSTN). The service area of a cellular network is divided into many smaller areas, called cells, each of which is served by a base station (BS) [1]. The BS is fixed and is connected to the mobile telephone switching office (MTSO), also known as the mobile switching center. An MTSO is in charge of a cluster of BSs and it is, in turn, connected to the PSTN. With the wireless link between the BS and MS, MSs such as cell phones are able to communicate with wireline phones in the PSTN. Both BSs and MSs are equipped with a 
transceiver. Figure 1 illustrates a typical cellular network, in which a cell is represented by a hexagon and a BS is represented by a triangle.

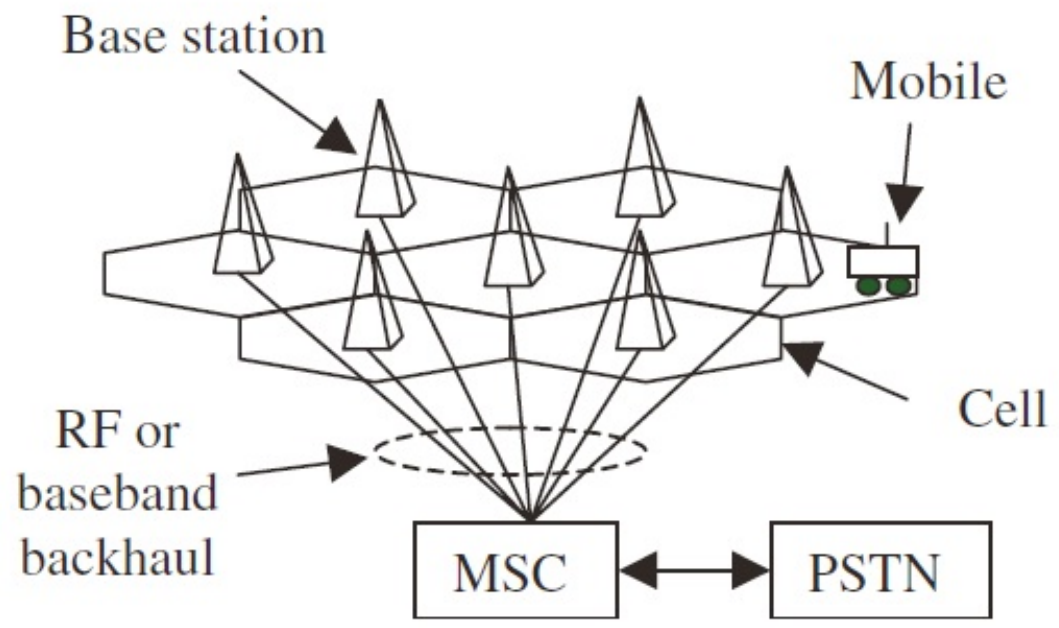

Figure 1.1: System architecture of a conventional cellular system

The success of todays cellular network is primarily due to the frequency reuse concept. The frequency spectrum allocated for cellular communications is very limited, however. Each BS (or cell) is assigned a group of frequency bands or channels. For the purpose of avoiding radio co-channel interference, the group of channels assigned to one cell must be different from the group of channels assigned to its neighboring cells. However, the same group of channels can be assigned to the two cells that are far enough apart such that the radio cochannel interference between them is within a tolerable limit.

Typically, seven neighboring cells are grouped together to form a cluster, as shown in Figure 1.2. The total available channels are divided into seven groups, each of which is assigned to a cell. In Figure 1.2, the cells marked with the same alphabet have the same group of channels assigned to them. Furthermore, the cells marked with different numbers must be assigned different groups of channels. 


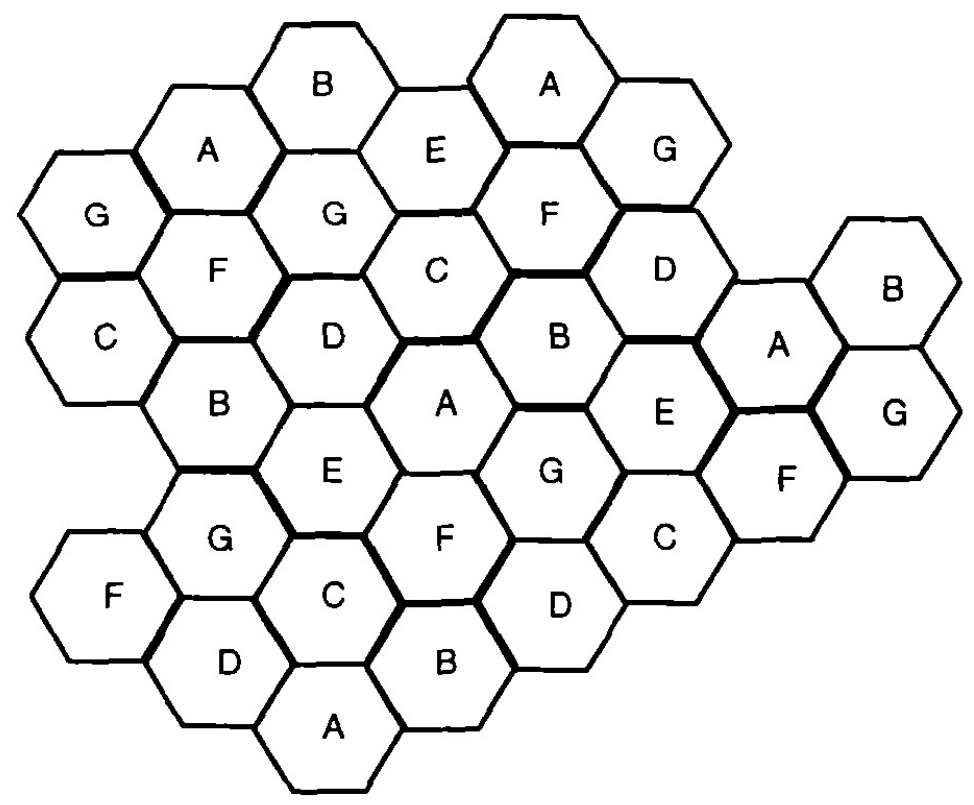

Figure 1.2: Frequency reuse

The channels assigned to a cell are generally used either for voice or for control. A voice channel is used for an actual conversation, whereas a control channel is used for setting up conversations. Both voice and control channels are further divided into downlink and uplink (forward or reverse). A forward channel is used to carry traffic from the BS to the MS, and a reverse channel is used to carry traffic from the MS to the BS. The channels assigned to a cell are shared by MSs located in the cell.

Every MS in the network has a home, which is the MTSO where the mobile user originally subsribed for wireless services and this location is stored in it. If an MS moves out of the home MTSO area, it is roaming. A roaming MS is required to register for services in the MTSO visited. An MS needs to be authenticated first before it can get any service by the network. The process of making a call is as follows. The MS first requests for service using a reverse control channel in the current cell. The request then needs to be granted, by the MTSO, a pair of voice channels assigned for the call, 
one for receiving and one for transmitting. Making a call to an MS is a little more complex, however. The call is first routed to the home MTSO or the visited MTSO if it is roaming. The MTSO needs to know the cell in which the MS is currently located. Here, the concept of location management comes in to locate the residing cell of an MS. Once the MTSO knows the residing cell of the MS, a pair of voice channels assigned in the cell for the call.

If a call is in progress when the MS moves into a neighboring cell, the MS needs to get a new pair of voice channels from the BS to the neighboring cell so the call can be continued. This process is known as handoff.

Cellular networks offer a number of advantages over other solutions :

- Increased Capacity: The increased capacity in a cellular network, compared with a network with a single transmitter, comes from the fact that the same radio frequency can be re-used in a different area for a completely different transmission, which is one of the key characteristics of it.

- Reduced Interference from Other Signals : In order to distinguish signals from several different transmitters, frequency division multiple access (FDMA) and code division multiple access (CDMA) were developed. In FDMA, the transmitting and receiving frequencies used in each cell are different from the frequencies used in each neighbouring cell. In CDMA, spread spectrum techniques are used by which a signal generated in a particular bandwidth is deliberately spread in the frequency domain, resulting in a signal with a wider bandwidth.

- Larger Coverage Area: The concept of dividing the land area into cells and strategically placing base stations in them, results in a larger coverage area as compared to other alternate solutions and is a significant feature of cellular networks. 
- Reduced Cost: Although the cost of deploying the BSs and setting up the network is substantial, taking into account the advantages that cellular networks offer over alternate solutions, the cost of operation is much less considering its use in the long term.

\subsubsection{Challenges in Cellular Networks}

Although cellular networks present many benefits, there are some inherent challenges and limitations on coverage and cell capacity. In addition to this, they also suffer from the problem of dead spots and then there is the problem of limited capacity which gives rise to the hot spot problem as described below. Lastly, there is the issue of radio resource utilization, which is the focus of our research in this thesis.

We discuss these limitations and issues as follows before narrowing down on the problem of non-uniform user distribution that causes a severe imbalance of load in the system.

- The dead spot problem : Although, mobile users are within the communication range of the $\mathrm{BS}$, there are still some areas where coverage is not available at all. These areas are often referred to as dead spots such as indoor environments and underground areas like basements.

- Limited capacity : In cellular networks, the capacity of a cell is limited by the number of channels allocated to the cell. The larger the number of channels, the greater the number of users that can be served. The number of channels is limited by the available frequency spectrums and by the frequency reuse factor [3]. A smaller cell size allows higher frequency reuse and, thus, a higher capacity can be achieved. The cell capacity is not only limited by the available frequency spectrums, but also by the interference among mobile nodes and BSs. The higher the interference, the lower the cell capacity is.

- The problem of hot spots : Due to the limited capacity, mobile users 
tend to experience higher call blocking, i.e., call requests are denied, in dense areas known as hot spots, such as downtown areas and amusement parks. This is because of the fact that, in hot spots, there are more mobile users than the number of available channels.

- The issue of radio resource utilization: The hot spot problem in turn raises the issue of radio resource utilization. While there are not enough channels or capacity in a hot spot for serving mobile users, the cells neighbouring the hot spot may still have available channels, leaving system resources under-utilized.

- Limitations on coverage: the coverage of cells is limited by the communication range or transmission power of the BS. Mobile users, which are outside the coverage of the BSs, are not able to access the networks due to this.

\subsubsection{Issue of Non-Uniform User Distribution}

One of the major performance degradation factors in cellular networks, which is the focus of our research in this thesis, is the issue of non-uniform user distribution due to received signal strength indicator (RSSI) based user association [3]. In cellular networks, a mobile station (MS) generally scans and associates itself with the base station (BS), which yields the maximal RSSI obtained by reading the power of the preamble signal, while being oblivious to the load of the BS. As the distribution of MSs are, typically, not even, some BSs tend to suffer from heavy load while the BSs adjacent to them may carry only light load, leading to under-utilized resources in the system.

In this kind of scenario, the boundary or cell-edge users in the network suffer the most from low throughput due to this uneven distribution which severely hampers the performance of the network. In order to alleviate this problem, new schemes need to be investigated that take into 
account the load of BSs, in addition to the conventional association metrics.

\subsection{Thesis Objective}

The main objective of this thesis is to explore the applicability of the preamble code sequence, which is used mainly for cell identification, as an implicit load indicator, towards the goal of designing distributed load balancing schemes to tackle the problem of imbalanced load due to non-uniform user distribution in cellular networks.

The motivation to explore and use preamble sequences comes from the fact that, with the growth of wireless subscribers, the service providers now face the challenge of how to maximize their network capacity with their existing infrastructure, and preamble sequences are already employed by almost every network for the pupose of cell identification, synchronization or channel measurement, thereby justifying the basis for our work. Following are the goals that we aim to acheive that can be classified as sub-objectives :

- Minimize the traffic load variances to achieve load balancing: The main goal, as stated forehand, is to develop schemes that consider not only the conventional metric RSSI for user association, but also the traffic load or congestion at the BS, allowing users to make a more intelligent decision on which BS to associate with. This would result in minimizing the variation in traffic load to achieve a well-balanced network in terms of load.

- Maximize overall network throughput and capacity: Once we are able to achieve the goal of load balancing, we should be able to witness an increase in the overall network and capacity as a result. In addition to this, we aim to develop ways to focus on the boundary or cell-edge users, which are the major targets of every load balancing scheme, 
to effectively reduce the deterioration in cell edge throughput, by getting more boundary users to participate in load balancing.

- Maximize Jain's fairness to achieve fairness among the mobile users : Fairness is a major criteria in evaluating how well the resources are being shared among the users equally. Therefore, our schemes should yield good results under popular fairness measures such as max-min fairness and proportional fairness and evaluation indexes like Jain's fairness index.

- Maximize robustness to perform well under different environments : Our schemes should be able to perform well under different channel conditions and be robust.

\subsection{Thesis Contributions}

In this thesis, we propose the following schemes to address the problem of non-uniform user distribution due to conventional RSSI-based association and confirm the applicability of the preamble code sequence as an implicit load indication tool for load balancing in cellular networks.

- Auto-correlation based implicit load indication scheme [4] modifies the preamble code sequence and proposes the auto-correlation operation as a way to decipher the load at a BS. This scheme enables the MSs to make an intelligent decision while choosing a BS for service, by not only considering the RSSI, but the level of load at the BS.

- Cross-correlation based implicit load indication scheme [4] expolits the cross-correlation property among preamble sequences to implicitly obtain the load state of BSs for load balancing. This enables the users while joining the network to perform smart cell-site selection based on the level of congestion at the BS along with the conventional association metrics. 
A part of this work titled "Utilizing the Inherent Properties of Preamble Sequences for Load Balancing in Cellular Networks" has been accepted by the 7th International Conference on Intelligent Sensors, Sensor Networks and Information Processing (ISSNIP 2011), Adelaide, Australia, Dec 6-9, 2011.

\subsection{Thesis Organization}

- Chapter 2 - Related Work gives a brief overview of the various load balancing philosphies that exist in the literature along with the recent research work on load balancing using preamble sequences.

- Chapter 3 - System Model describes the employment of the IEEE 802.16e WiBRo system for the purpose of illustration and gives a brief description of our concept of utilizing preamble sequences for load balancing including the defintions.

- Chapter 4 - Auto-Correlation based Implicit Load Indication Scheme describes the design and implementation of the auto-correlation based implicit load indication scheme.

- Chapter 5 - Cross-Correlation based Implicit Load Indication Scheme describes the design and implementation of the cross-correlation based implicit load indication scheme.

- Chapter 6 - Simulation Results and Discussion presents the results obtained through simulations with a breif discussion on the achievements.

- Chapter 7 - Conclusion and Future Work talks about a breif summary of what we have acheived in our research and their potential along with the road ahead. 


\section{Chapter 2}

\section{Related Work}

\subsection{Load Balancing in Cellular Networks}

Traditionally, load balancing has been widely studied for the efficient utilization of the network resources. By doing so, users can enjoy better throughput while the operator may support more subscribers by utilizing the deployed network resources more efficiently. In this section, we provide an overview of the load balancing and capacity maximization schemes that exist in the literature.

\subsubsection{Channel Assignement Schemes}

There are three main channel assignment strategies proposed in the literature [2]. The first one is fixed channel assignment (FCA) strategy, wherein each cell is allocated a predetermined set of voice channels. If all the channels in a cell are occupied, any new calls or handover requests within the cell will be rejected. In variants of the FCA strategy, a cell is allowed to borrow channels from a neighboring cell if all of its channels are already occupied. The mobile switching center (MSC) supervises such borrowing procedures and ensures that borrowing of a channel will not affect any of the calls already in progress in the cell which is lending. Simplicity is 
one of the main advantages of such a scheme along with frequency reuse maximization, as opposed to the other schemes which have complex procedures [2].

The second one is the dynamic channel assignment (DCA) strategy, in which voice channels are not permanently allocated to the cells [2][5][6][7]. Everytime a call request is made, the serving base station requests a channel from the MSC, instead. The switch then allocates a channel to the requesting cell following an algorithm that takes into account the likelyhood of future call blocking within the cell, the frequency of use of the candidate, the reuse distance of the channel, and other cost functions. Although DCA schemes improve the call blocking probability of the system substantially, for high traffic intensity, the computational load on the system increases greatly.

The third type is hybrid channel assignment (HCA), which is a blend of the concepts of fixed and dynamic channel assignement schemes. This basically means, there are channels in a global pool for use in case there is a shortage, in addition to the fixed set of channels assigned to each cell [8].

To conclude, although the objective of all the schemes is better utilization of the available channels, causing reduced call blocking probability, most of the schemes do not consider the non-uniformity of channel demand, which is the variation of channel demand from time to time and/or area to area.

\subsubsection{Dynamic Load Balancing Schemes}

The dynamic load balancing schemes proposed in the literature can be broadly classified into two groups:

i) Strategies based on channel borrowing from cooler cells (lightly loaded) such as simple borrowing [8]-[9], channel borrowing without locking (CBWL) [10], load balancing with selective borrowing (LBSB) [11], etc.

ii) Strategies based on traffic transfer to cooler cells such as directed 
retry [12]-[13], hierarchical macrocell overlay systems [14], etc.

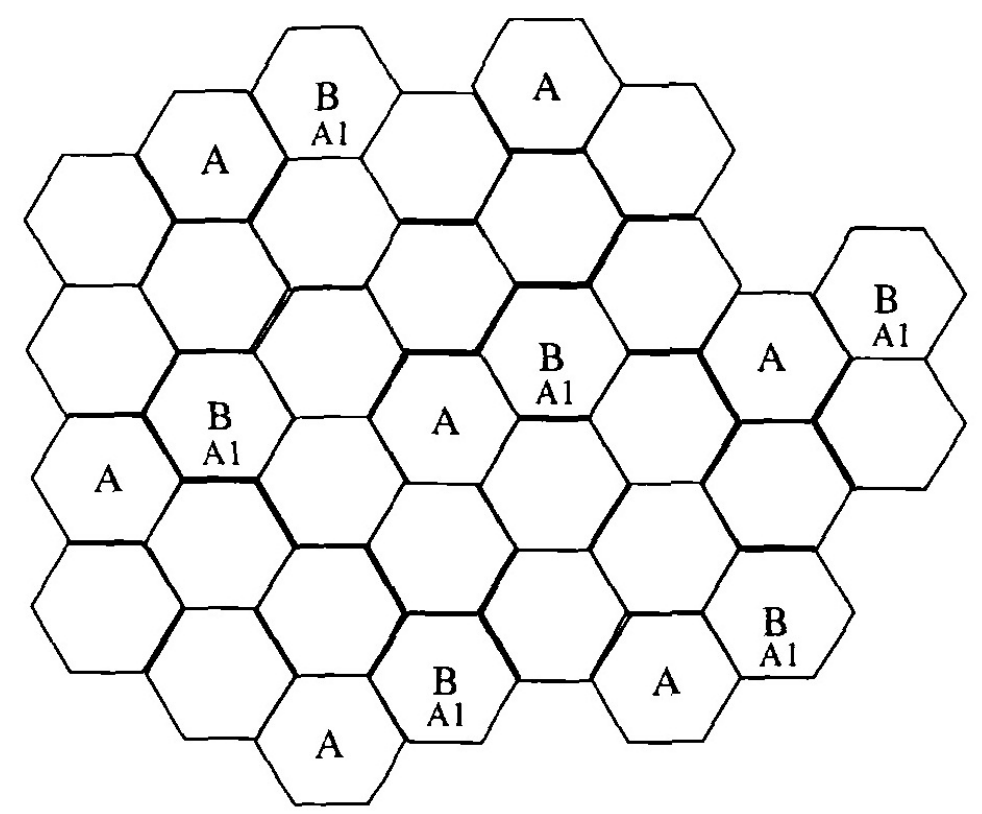

Figure 2.1: Channel borrowing without locking (CBWL).

In simple borrowing scheme, every cell is assigned a fixed set of channels and a seperate set of channels that are allowed to be borrowed by the neighboring congested cells [8]-[9]. When a channel is borrowed, the co-channels within the reuse distance are locked to avoid co-channel interference.

In CBWL, the use of borrowed channels with reduced transmission power is proposed, when the set of available channels in a cell gets exausted [10]. This is done to avoid interference with other co-channels. Taking advantage of transmission power control, however, to induce handoff's into less loaded cells and reducing it, may decrease the link capacity for others, who still remain in the cell.

There are two types of CBWL: $i$ ) CBWL without channel arrangement (CBWLnR); and ii) CBWL with channel rearragement (CBWLCR). In CBWLnR, only the new call requests within the fraction of cellular area in 
which borrowed channels can be used (this area is limited due to reduced transmission power over the borrowed channels) are granted service using a borrowed channel. However, in CBWLCR, even if the new call request is not in the channel-borrowing area, it can still get service if there is at least one active user within the channel-borrowing area. In this case, the active user will borrow a channel from a neighboring cell and start transmission at a reduced power and the channel released by that user will be assigned to the new user requesting service.

In selective channel borrowing [11], load balancing is achieved by a structured borrowing mechanism where a hot cell can borrow a fixed number of channels only from adjacent cells in the next outer ring. In this way, unused channels of the cold cells are moved to the hot spot area. This mechanism reduces the amount of interference between the borrower cell and the co-channel cells of the lender.

In directed retry scheme, the traffic load is shared in the overlap of the neighbouring cells. In this, there is no concept of borrowing channels from the neighboring cells, however, the subscribers who can communicate with more than one base station are moved from the congested cell to a neighboring cell [12].

In the hierarchical macrocell overlay system with microcells and overlaid macrocells [14], high traffic areas are covered by microcells while overlaying macrocells cover low-traffic areas and provide overflow groups of channels for clusters of microcells. In other words, the excess traffic of the microcells is served by the overlaying macrocells. As a consequence, macrocell overlays inherently achieve dynamic load balancing by transferring the otherwise blocked microcell users to the macrocells.

\subsubsection{Schemes based on Association Control}

The most widely implemented association procedure uses the RSSI as a metric for association, which causes non-uniform user distribution across 
cells, as the load of the BS is not considered. This often causes the entire network to be under-utilized.

To combat this, several studies [15]-[21] have proposed a variety of association metrics instead of using the RSSI as the sole association criteria. These metrics typically consider factors such as, the number of users currently associated with a BS, and the bandwidth that a new user will be able to get if it is associated with a BS [15]-[16]. Balachandran et al. [17] proposed to associate a user with the AP that can provide a minimal bandwidth required by the user. If there exist many such APs, the one with the strongest signal strength is selected.

Velayos et al. [18] introduced a distributed load balancing architecture where the load of an AP is defined as the aggregated downlink and uplink traffic through the AP. Kumar et al. [19] proposed an association selection algorithm which is based on the concept of proportional fairness to balance between throughput and fairness. Most of these work heuristically determine only the association of newly arrived users with some exceptions [20] [21]. Tsai and Lien [20] proposed to reassociate users when the total load exceeds a certain threshold or the bandwidth allocated to users drops below a certain threshold. An on-line scheme that periodically optimizes the user-AP association has also been proposed [21].

\subsubsection{Relay Assisted Traffic Transfer Schemes}

Recently, there has been a lot of interest in the usage of relays and relaying techniques to expand coverage and capacity in cellular networks. Researchers have looked at the problem of load balancing from the perspective of relaying and have proposed schemes to induce user handoff's from heavily loaded cells into adjacent cells that may be lighly loaded.

iCAR [35] and PARCelS [36] were the first two load balancing schemes introduced for balancing the load among cells through relaying. In iCAR, low cost limited mobility, ad-hoc relay stations (ARSs) are placed in hot 
spots for relaying traffic out of the hot spots. This strategy is still costly and not flexible enough to handle the highly dynamic load situation in $3 G$ networks. PARCelS uses mobile nodes for relaying. When a BS is congested, mobile nodes search best routes to other non-congested cells. Route information is forwarded to BSs for selection. This strategy requires considerable routing overhead in mobile nodes and does not take advantage of the presence of powerful BSs. In addition, both schemes do not take into account the load balancing among relaying nodes which could greatly affect the load balancing performance.

Some other schemes have also been introduced to increase the capacity in cellular networks with the schemes based on the same idea and differing only in implementation.

Mobile Assisted Call Admission (MACA) [23], is a dynamic load balancing scheme proposed to improve call blocking probability performance in cellular networks with the idea of forwarding the excess traffic of a "hot" cell to its "cooler" neighboring cells via mobile agents in the network. For the purpose of forwarding the call, either relay channel can be used, in-band (i.e., cellular band) channel or out-of-band (i.e., ISM-band or any other band other than cellular band) channel. In the case of in-band MACA, a portion of the fixed channels assigned to each cell is saved for forwarding calls. Whereas, in out-of-band MACA, the mobile agents use channels from a frequency band other than the cellular band such as the ISM-band to forward calls.

In Multihop Cellular Networks (MCN) [22], the range of the BSs and mobile devics are reduced and mobile users within the same cell are able to communicate with each other over multi-hops, and on the other hand, mobile users in different cells need to forward their traffic via BSs. The objective in this scheme is to increase the capacity of the cellular network by replacing single-hop communication with multi-hop between the BSs and mobile users. 
In Unified Cellular and Ad hoc Network (UCAN) [24], instead of improving the call blocking probability, the use of ad hoc wireless connection to enhance a mobile user's access to the 3G cellular infrastructure is proposed. If the throughput is low (i.e., the direct wireless link from the BS to MS is bad), a multi-hop link from the mobile user to the BS is formed via other mobile users in the system that have better channel quality.

\subsection{Load Balancing in Wireless Local Area Net- works}

Many of the schemes based on association contol described previously also apply to WLANs, as there is not much fundamental difference when it comes to applying load balancing between WLANs and celluar networks. The only difference is in the channel allocation schemes which are not applicable to WLANs as each AP normally uses one channel and channel allocation is fixed.

In WLANs, generally the concept of Cell Breathing [37]-[38], sometimes also referred as dynamic cell re-sizing, has been used for load balancing, in which the geographical area covered by the cell tower is constantly changed by controlling the transmission power of an AP's beacon packets. The heavily loaded cells shrink their coverage area, and the lightly loaded cells expand their coverage area to attract clients previously associated with the heavily loaded cells.

Both [26] and [27] take advantage of transmit power control to induce the users handoff into less loaded cells. However, reducing the transmission power of BS may decrease the link capacity for other users, who still remain in the cell with reduced transmit power. In order to deal with this problem, S. Das et al. [29] proposed to control the power of common pilot channel without changing the power allocated to data channel. However, this is not practical considering the recent trend that the transmission rate 


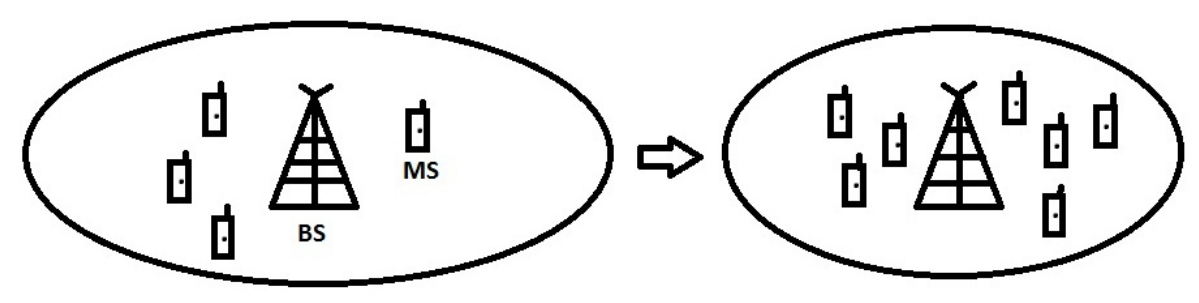

Figure 2.2: Cell breathing (decrease in coverage area with increase in subscribers)

is adapted via adaptive modulation and coding (AMC) according to the channel condition, which is typically known from measuring the power of common pilot channel.

\subsection{Recent Research Work}

In a recent study by $\mathrm{Na}$, et al. [30], it has been shown that preamble sequence can be used as an implicit load indication tool. The work exploited comparative high and low cross correlation among certain sets of preamble sequences, to indicate similarity in terms of lightly loaded and heavily loaded state of the BSs for cell selection. However, the proposed scheme fails to address all the permutations of BS loads, and in situations where certain PN sequences outside the set would yield high correlation with sequences within it.

The scheme also does not consider the case of two heavily loaded and one lightly loaded cell situation, and although this may not be a degradation and the MS would select a BS based on the conventional received signal strength (RSSI) association, it exposes the limitations of the scheme. We, therefore, propose an alternative approach in our cross-correlation based load balancing scheme to use preamble sequences in such a way 
that makes the implicit indication absolute as opposed to relative, thereby, removing the inherent difficulties.

\subsection{Summary}

In this chapter, a brief overview of the all the load load balancing schemes proposed for cellular networks, was presented. We discussed the various channel assignment schemes which allocate the channels to cells using different strategies with the aim of maximizing the utilization of the available channels for an increase in capacity and performance.

We talked about the dynamic load balancing schemes, wherein the capacity is increased via dynamic load/traffic transfer among cells. A brief note on load balancing schemes based on association control is also presented, in which, several schemes have been proposed that use different metrics to associate users with BSs in addition to the conventional RSSIbased association.

Schemes based on relay assisted traffic transfer are also discussed which use relays and relaying techniques to expand coverage and capacity. We have also presented load balancing schemes designed for WLANs, where the cell size is dynamically changed for load balancing, a phenomina called as cell breathing, along with schemes relying on transmit power to induce user handoffs into cells which are comparatively less loaded.

Finally, recent research work into load balancing using preamble sequences was presented with its features and limitations. In the next chapter, we present our system model, where we present our concept of load balancing using preamble sequences. 


\section{Chapter 3}

\section{System Model}

\subsection{Definitions}

\subsubsection{Preamble Sequences}

Preamble sequences, also known as pseudo-noise sequences (PN sequences), are sequences that are deterministically generated and yet possess some properties that one would expect to find in randomly generated sequences. They are called PN sequences because of their low autocorrelation for positive delays. This makes them nearly random, even though they are deterministic in nature.

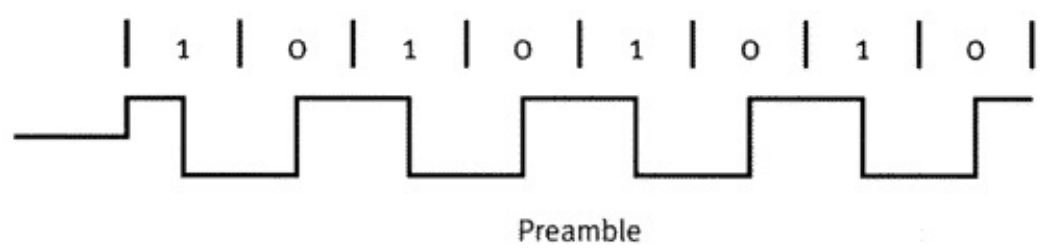

Figure 3.1: A sample pseudo-noise sequence

They are useful for cell identification in cellular networks because of their uniqueness, i.e. different PN sequences are highly uncorrelated with each other, but the cross correlation of a PN sequence with an identi- 
cal copy of itself produces a peak at the origin. The definitions of autocorrelation and cross-correlation are defined subsequently. Applications of PN sequences include signal synchronization, navigation, radar ranging, random number generation, spread-spectrum communications, multipath resolution, cryptography, and signal identification in multiple-access communication systems [39].

In the design of the preamble, the following must be taken into consideration. The preamble sequence for cellular systems should have a low peak-to-average power ratio (PAPR), which is is the ratio of peak signal power to the average signal power used for estimating the backoff required for an radio-frequency/microwave power amplifier to exhibit acceptable intermodulation distortion. Along with this, it should also possess good (low) cross-correlation and (impulse-like) auto-correlation characteristics all with the aim of serving well for the purpose of cell identification, time and frequency synchronization and channel measurement. Well-known preamble sequences for cellular systems include PN sequence, Gold sequence, and CAZAC sequence [46].

Various pseudo random codes are generated using LFSR (Linear Feedback Shift Register). The generator polynomial governs all the characteristics of the generator. For a given generator polynomial, there are two ways of implementing LFSR. Galois feedback generator uses only the output bit to add (in Galois field) several stages of the shift register and is desirable for high speed hardware implementation as well as software implementation.

The other one is the Fibonacci feedback generator which can generate several delays of sequences without any additional logic. Shift-register sequences having the maximum possible period for an r-stage shift register are called maximal length sequences or m-sequences. A primitive generator polynomial always yields an m-sequence. The maximum period of an $\mathrm{p}$-stage shift register can be proven to be $2^{p}-1$. The m-sequences has three important properties, i.e., balance property, run-length property and 
shift-and-add property. An MLS-generating system with a shift register of length 4 is shown in Figure 3.2. It can be expressed using the following recursive relation:

$$
a_{k}(n+1)= \begin{cases}a 0(n)+a 1(n), & k=3 \\ a_{k+1}(n), & \text { otherwise }\end{cases}
$$

where $\mathrm{n}$ is the time index, $\mathrm{k}$ is the bit register position, and + represents modulo-2 addition. As MLS are periodic and shift registers cycle through every possible binary value (with the exception of the zero vector), registers can be initialized to any state, with the exception of the zero vector.

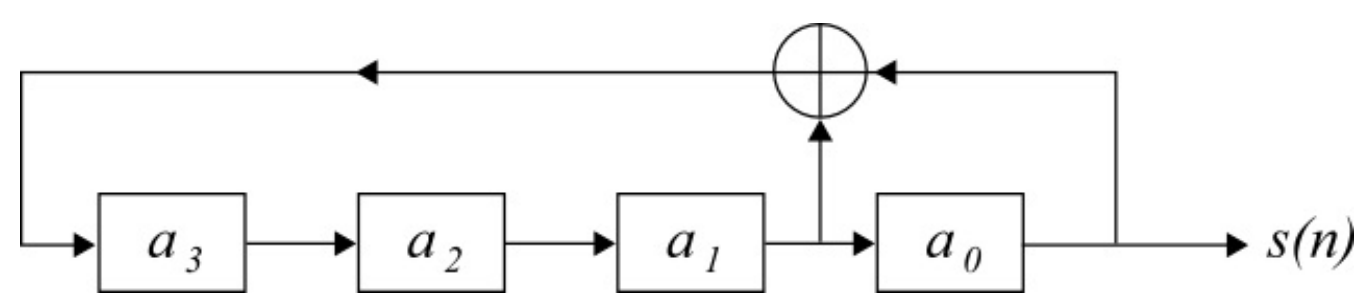

Figure 3.2: M-Sequence generator

\section{Polynomial Interpretation}

A polynomial over GF(2) can be associated with the linear feedback shift register. It has degree of the length of the shift register, and has coefficients that are either 0 or 1 , corresponding to the taps of the register that feed the xor gate. For example, the polynomial corresponding to Figure 3.1 , is $x^{4}+x^{1}+1$. A necessary and sufficient condition for the sequence generated by a LFSR to be maximal length is that its corresponding polynomial be primitive.

\subsubsection{Fairness in Wireless Networks}

Fairness is an important property of a resource allocation algorithm. Fairness measures or metrics are used in network engineering to determine 
whether users or applications are receiving a fair share of system resources. When network resources are insufficient to satisfy demand, they should be divided fairly among network users [42]. There are two main approaches to model fairness in resource allocation : max-min fairness [43] and proportional fairness [44]. Max-min fairness is achieved by allocating available resources to the maximum extent possible to the disadvantaged users, while not necessarily wasting resources. On the other hand, proportional fairness approaches the problem from another perspective; proportional fairness is realized when an objective function is maximized that represents the overall utilization of all users while respecting the total available resource constraint.

Both proportional fairness and max-min fairness possess optimality properties and are Pareto optimal (i.e., if one cannot increase the assignment to one source $i$ without strictly decreasing an assignment to another source $j$ ) and can be implemented under the convex optimization [45].

While considering the fact that in a wireless environment, because of the random channel variations, we should be able to distinguish between ef fort (radio resource given to user) and outcome (actual useful throughput achieved by the user). Normally, effort will be equal to the outcome, but in a wireless environment, they can be substantially different. Both max-min and proportional fairness can be defined based on either effort or outcome.

Due to the time varying nature of wireless channels, fairness is usually defined and implemented over a period of time. Based on the length of the period, two kinds of fairness are considered : short term fairness and long term fairness. Short term fairness means the ability to provide equal allocation of resources to all active connections over short time scales; and long term fairness is the opposite, and is concerned with the amount of resources assigned over a longer time-scale. 


\subsection{Utilizing Preamble Sequences for Load Bal- ancing}

In a cellular network, every BS is assigned a unique preamble sequence. All the MSs in the network are embedded with the all the preamble sequences that exist in a particular technology and are cognizant about the assignment of the sequences to BSs. Therefore, when they enter the network and recieve the preamble signals from BSs, the simply correlate these sequences with the all the sequences they know about to find a match and identify the cell. This process if known as Cell Identification.

In this thesis, we configure the preamble sequences to implicitly indicate the load experienced by the BS they are associated with, to MSs seeking association and service. This allows the MSs to make decisions that are more efficient in terms of fairness, depending on the quality of service (QOS) measure we take as a criteria. We use the properties of high autocorrelation and low cross-correlation to define certain metrics first, which will be used to sort all the preamble sequences of the system.

Once the sequences have been sorted, we link the load information to the value of the metric associated with the preamble sequence. This is done by mapping the sequences to BSs according to the metric values and the load experienced by the BS. This implies, for a highly loaded BS $b$, the corresponding metric defined on $b$ 's preamble sequence $\vec{p}_{b}$, would have a higher value and for a relatively lightly loaded BS, the value would be small.

We consider the following preamble configurations towards the design of our schemes:

- A sequence $\vec{p}$ formed by repetition of a part of a preamble sequence on autocorrelation forms a number of peaks in the autocorrelation 
plot which will be proportional to the load experienced by the BS corresponding to this sequence $\overrightarrow{p^{\prime}}$.

- A preamble sequence of a lightly loaded BS will be highly correlated with a particular code sequence $\vec{p}_{0}$ (known to both the MS and the controller) and vice-versa.

Using the above described metrics, the sequences corresponding to the BSs are sorted and assigned in a predefined order as shown in Figure 3.3 for the auto-correlation based load indication scheme and Figure 3.4 for absolute cross-correlation scheme.

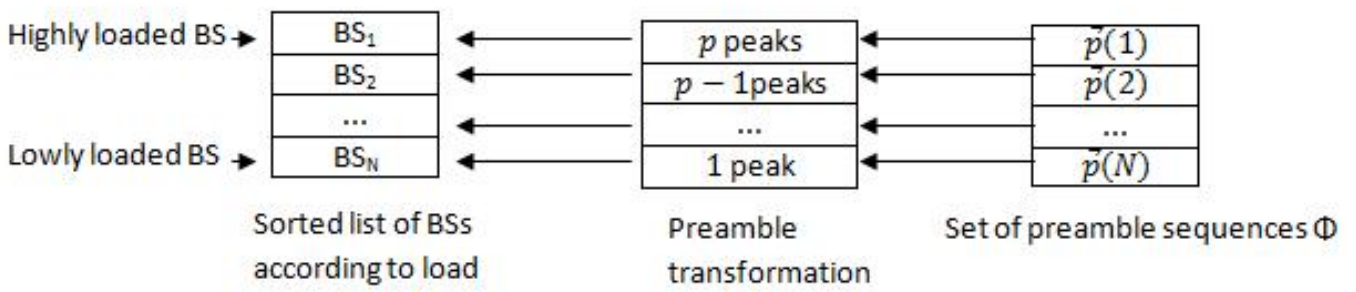

Figure 3.3: At central controller the metric is the number of peaks in the auto-correlation plot.

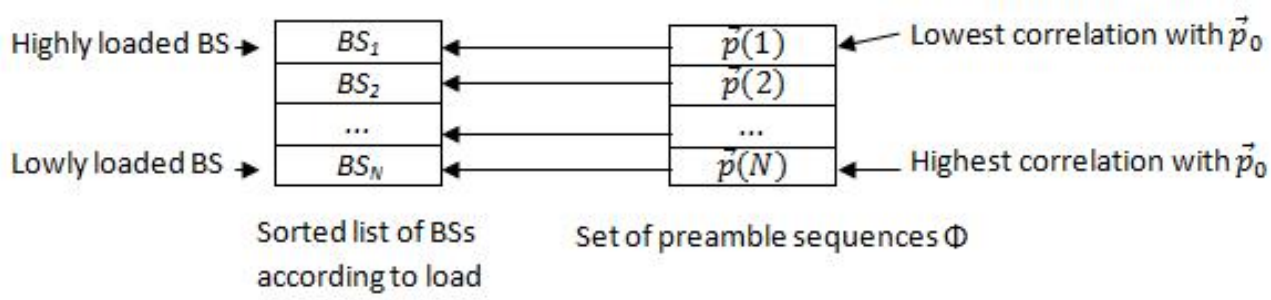

Figure 3.4: At central controller the metric is the value of cross-correlation with particular sequence. 


\subsubsection{Load Balancing parameter}

In each of the above mentioned configurations, we propose two algorithms which work in parallel at the MS and at the central network controller. For the algorithms to implement the load balancing feature, we propose a design parameter $r$ which is basically a measure of how much greater the strongest RSSI is over the next strongest RSSI in the system, when our algorithm stops coming into effect. This physically implies the measure of area near the border between two cells where load balancing comes into effect. As $r$ increases, more area near the boundary is considered and vice-versa. Here, $r=1$ will be the limiting case, where the ratio occurs at the cell boundary.

$$
\alpha=\frac{R S S I_{\text {strongest }}}{R S S I_{\text {nextstrongest }}}=1
$$

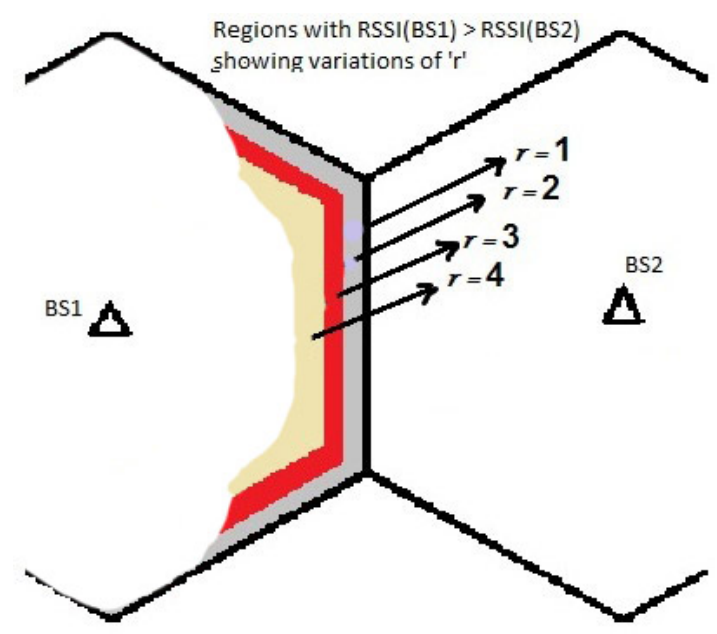

Figure 3.5: A two cell model showing the concept of " $r$ ".

From this limiting case onwards, when $r>1$, larger areas near the boundary are considered for load balancing. Additionally, the conventional RSSI based cell selection can be invoked by setting $r=0$, when 
the algorithm skips the load balancing steps and adopts the conventional method using RSSI since $\alpha$ is always positive.

Thus, when $r=0$, the condition for $\alpha<0$ will never be satisfied as can be seen later in the MSCD_AutoCorr and MSCD_CrossCorr algorithms, and can be better understood from Figure 3.5 above.

\subsection{System Model}

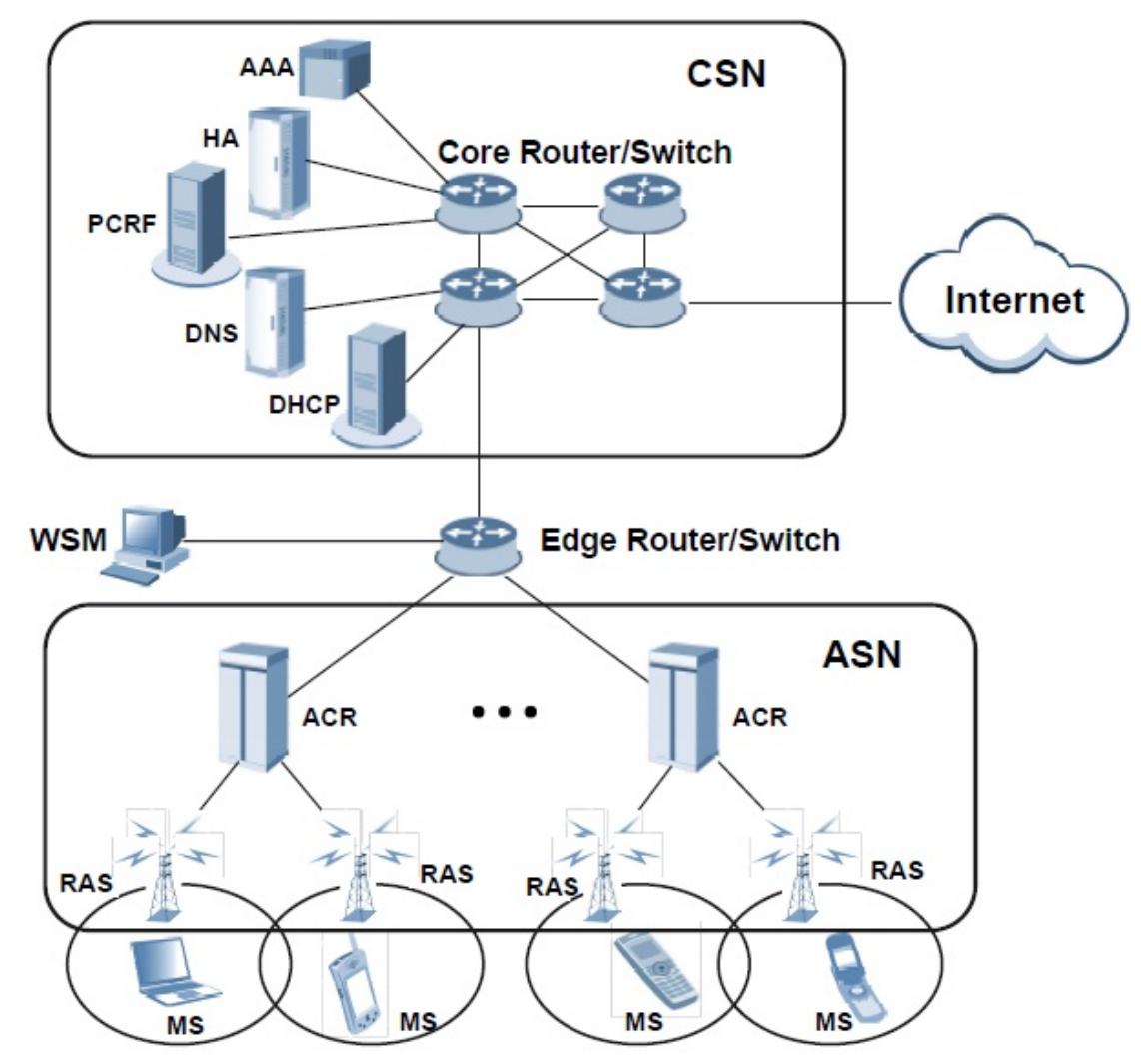

Figure 3.6: Architecture of the Korean WiMax Broadband [30]

For the illustration purpose, we in this thesis, consider the IEEE 802.16e/Mobile 
WiMAX system. Figure 3.6 shows the network architecture of WiBro (Wireless Broadband) system, which is a Korean version of Mobile WiMAX.

The WiBro network is composed of MSs, access network (ASN) and connectivity service network (CSN). Among the network components, a WiMAX system manager (WSM) is devised to manage multiple radio access stations (RASs or BSs) and access control routers (ACRs or ASN$\mathrm{G} / \mathrm{W})$ for the purpose of network optimization. ACR is the central system of the Wibro network, which connects the CSN and RAS. It enables multiple RASs to internetwork with CSN and IP networks and sends and receives traffic between the external network and MS. Therefore, it can be used as the centralized controller over WiBro system. We also emphasize that our proposed schemes can be used in any other wireless system, which employs preamble code sequences.

The IEEE 802.16e specification [31] defines 114 pseudo noise (PN) sequences to be used as the preamble sequences in the $1 \mathrm{k}$ Fast Fourier Transform (FFT) mode. These sequences are 284 bits long, and have the characteristic properties that make them appealing for applications like cell identification and synchronization.

In our schemes, we work with decisions involving cross-correlation between the PN code sequences, as the average cross-correlation between the different $\mathrm{PN}$ code sequences have been found to be ranging from a minimum of 0.04 to a maximum of 0.16 [30]. Also, a recent study [33] shows, it is possible to use just a part of the whole PN code sequence to practically achieve the goals of synchronization and cell identification, mainly because of this cross-correlation property among different sequences.

In a practical cellular network, a station generally selects the BS, which yields the maximal received signal strength indicator (RSSI) obtained by reading the preamble code sequence. In a model where $\mathcal{B}$ is the set of all the base stations, $g_{b s}$ is the channel gain from BS $b$ to an MS $s, P_{b}$ is the transmission power of $\mathrm{BS} b, \vec{p}_{b}$ is the preamble code sequence corresponding to base station $b$, and $\vec{x}_{s}(t)$ is the signal received by an MS $s$ at time $t$ 
from all BSs; the decision made by the MS $s$ is given in Eq. 5.1, where $\hat{b}$ is the chosen BS.

$$
\hat{b}=\arg \max _{b \in \mathcal{B}} R S S I_{s}(t, b)
$$

RSSI refers to the signal component of base station $b$, and $\vec{p}_{b} \in \mathcal{P}$ refers to the preamble sequences, such that $\mathcal{P}$ is the set of all BS preamble sequences.

$$
R S S I_{s}(t, b)=\vec{p}_{b} \cdot \vec{x}_{s}(t)
$$

In addition, the $k^{\text {th }}$ sequence in the set can be referred to as $\vec{p}(k)$ and will be used in the later sections.

$$
\vec{x}_{s}(t)=\sum_{b \in \mathcal{B}} g_{b s} P_{b}(t) \vec{p}_{b}
$$

\subsection{Summary}

In this chapter, we have presented a brief definition of preamble sequences, how they are generated, and their applications, along with a note on fairness in cellular networks. We introduced our proposed concept of how preamble sequences can be utilized for load balancing with a model/design parameter to target the boundary users. Finally, we presented our system model, and used the IEEE 802.16e/WIMAX system for illustration, with a note on its architectural components and how we use the preamble sequences in it for load balancing. In the next chapter, we present our proposed auto-correlation based implicit load indication scheme with the algorithms discussing how it is realized. 


\section{Chapter 4}

\section{Auto-Correlation based Implicit Load Indication Scheme}

\subsection{The Auto-Correlation Property}

Auto-correlation is defined as the cross-correlation of a signal with itself. It is the similarity between observations as a function of the time separation between them. It is a mathematical tool for finding repeating patterns, such as the presence of a periodic signal which has been buried under noise, or identifying the missing fundamental frequency in a signal implied by its harmonic frequencies [40]. It is often used in signal processing for analyzing functions or series of values, such as time domain signals.

In the case of m-sequences, there are two types of auto-correlation : periodic auto-correlation and aperiodic auto-correlation. If the synchronization window is only one period long or less, then the correlation is aperiodic, otherwise it will be always be periodic if it is any longer. As an example, we consider the auto-correlation of a sine wave with a frequency of 1000 $\mathrm{Hz}$ (Hertz) and a sampling frequency of $8000 \mathrm{~Hz}$. In the auto-correlation plot, the peaks are clearly visible which are used for the applications mentioned above and in this thesis, we use them to indicate the level of load to the MS while cell selection. 

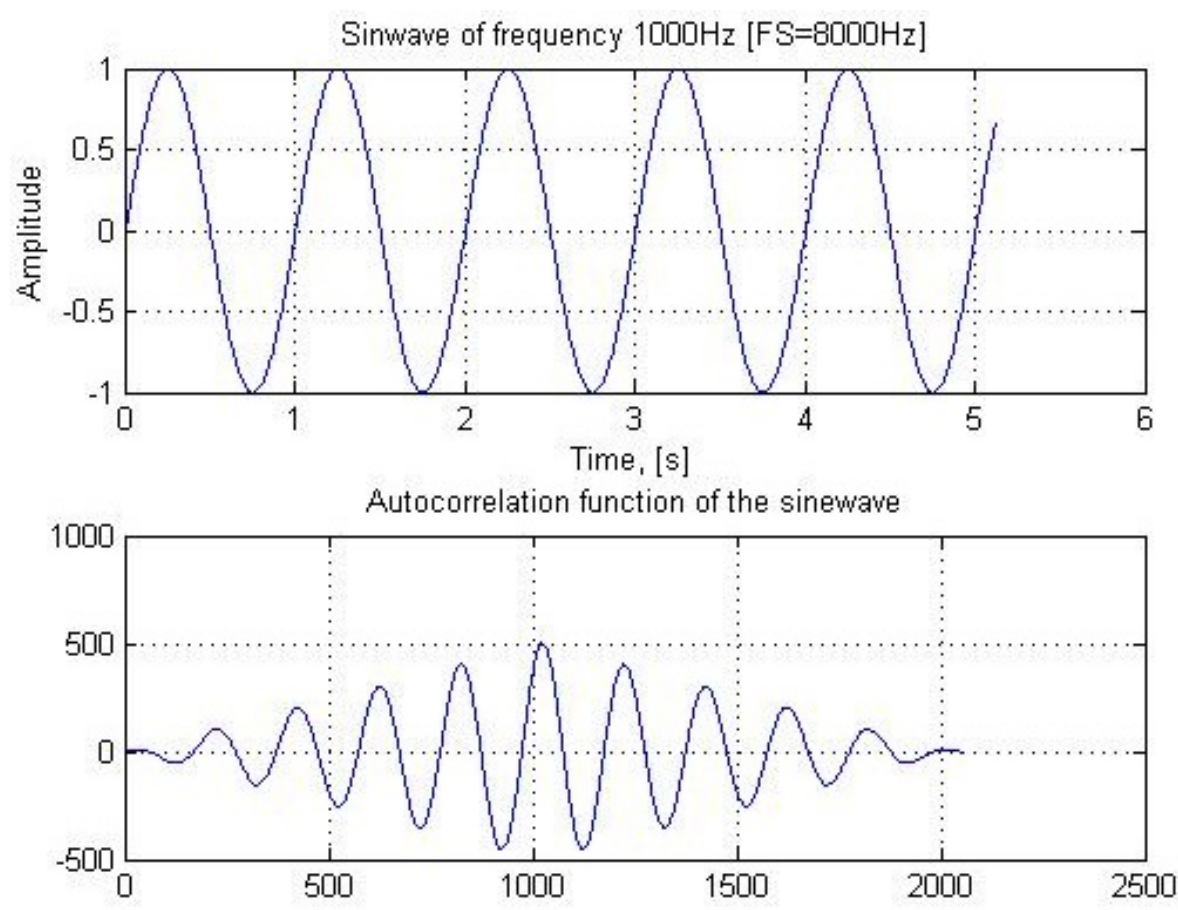

Figure 4.1: Auto-correlation function of the sinewave

In this scheme, we propose two algorithms working side by side to provide BS load indication using the preamble sequence. In this method, we modify the preamble code sequence and propose the autocorrelation operation as a way to decipher the load in a BS. This approach works at two levels: at the MS and at the central network controller.

Here, $\mathcal{B}$ is the set of all BSs and a particular BS is $b$ with load $l_{b}$. First, the central controller creates a list of BSs sorted in order of their loads. Then, we define $p$ intervals of the load value which can be denoted by $L_{1}, L_{2}, \ldots L_{p}$. Thus, the load values $l_{b}$ pertaining to the base station $b$ fall in one of these ranges.

In a general case where the preamble sequence length is $M$ bits, the autocorrelation plot of the sequence will give a peak at the origin and negligible values for all other delays. This is a characteristic feature of the PN sequences due to their pseudo random nature. We modify the PN se- 


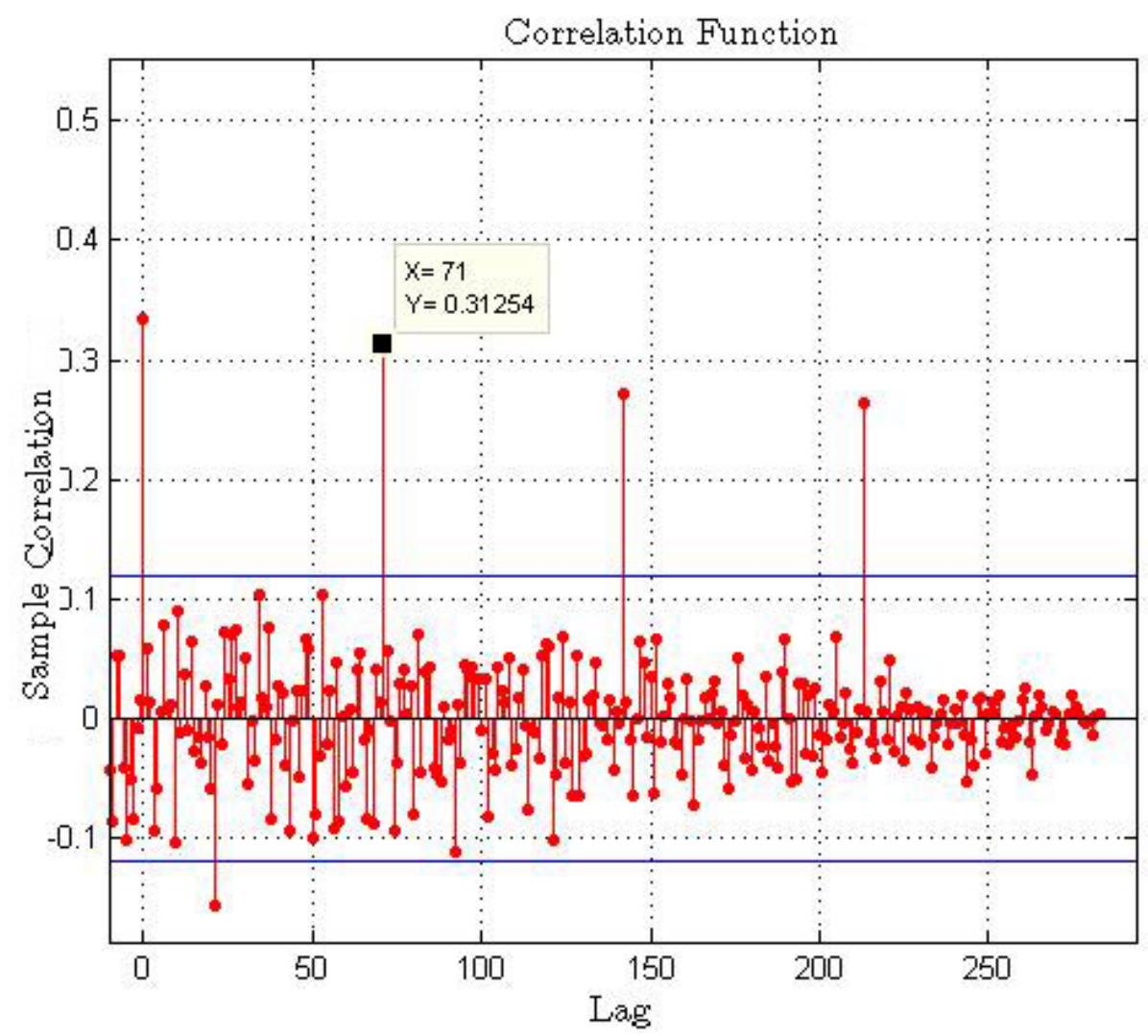

Figure 4.2: Auto-correlation plot of a preamble of length 284 bits made with 4 repeated sequences of length 71 .

quence by selecting the first $\frac{M}{j}$ bits of the PN sequence and then repeating it $j$ times where $j=\arg \left\{L_{j}\right\}$ and this will grouped into one of $L_{j}$. This will give us a sequence of $M$ bits which would be periodic in the time domain and would have $j$ periods in the $M$ bit length.

Figure 4.2 shows the auto-correlation plot of a preamble of length 284 bits which is made up of four repeated preamble sequences of length 71 .

After this operation, the autocorrelation plot for the modified preamble code shows $j$ peaks corresponding to the number of repetitions in the $M$ bit sequence. We propose that this value $j$ be the metric which we use for load indication of a particular BS. Hence, the more the repetitions in 
the preamble sequence, the more will be the number of peaks in the autocorrelation plot and that would indicate a more loaded BS.

This modification to the preamble code sequence by introducing periodicity in the time domain does cause concerns over its effect on cell identification and synchronization which the preamble sequence actually has to fulfill. These are valid concerns as due to the periodic nature of the modified preamble, it exhibits lower separation of magnitude between the autocorrelation peak and other values on the autocorrelation plot. These concerns have been addressed in a recent study [33], which shows that a considerably small part of the preamble sequence is practically sufficient in terms of achieving the required goals of identifiability and also synchronization. Larger number of repetitions would make the errors higher, but below a maximum number of allowed repetitions $p$, it would be practically sufficient. We now present two algorithms working side by side. The preamble modification algorithms works at the central controller and MS cell decision algorithm works at the user end.

\subsection{Preamble Modification Algorithm}

The modification of the preamble code would happen at the central network controller where it will assign the $j$ value (number of repetitions) to the BSs according to their loads.

This will be done by first identifying the maximally loaded BS in the set of BSs $\mathcal{B}$ (line 3). Then, the group number of the load value is extracted (line 4,5) and accordingly, the preamble sequence is modified (line 7 - 9) by taking the first $\frac{M}{j}$ bits of $\vec{p}$ and repeating it $j$ times (line 8). Thus, the BSs will then transmit using preamble sequences that have $j$ repetitions of the first $\frac{M}{j}$ bits of the original sequence. We refer to this as Preamble Modifying Algorithm (PMA). 


\begin{tabular}{|l|}
\hline Pseudo-code for $\operatorname{PMA}$ \\
\hline 1: procedure $\operatorname{PMA}(p, \mathcal{B})$ \\
2: while $\mathcal{B} \neq \emptyset$ do \\
3: $\dot{b} \longleftarrow \arg \max _{b \in \mathcal{B}} l_{b}$ \\
4: if $l_{\dot{b}} \in L_{j}$ then \\
5: $j \longleftarrow \arg \left\{L_{j}\right\}$ \\
6: endif \\
7: $\overrightarrow{p^{\prime}} \longleftarrow \overrightarrow{p^{b}}$ where $\overrightarrow{p^{\prime}} \in \mathcal{R}^{M}$ \\
8: $\overrightarrow{p^{\prime}} \longleftarrow\left[\overrightarrow{p^{\prime}}\left(\frac{M}{j}\right) \overrightarrow{p^{\prime}}\left(\frac{M}{j}\right) \ldots \vec{p}^{\prime}\left(\frac{M}{j}\right)\right]$ (repeated $j$ times) \\
9: $\overrightarrow{p_{b}} \longleftarrow \vec{p}^{\prime}$ \\
10: $\mathcal{B} \longleftarrow \mathcal{B} /\{\dot{b}\}$ \\
11: end while \\
12: end procedure
\end{tabular}

Table 4.1: Preamble Modification Algorithm (PMA)

\subsection{MS Cell Decision using AutoCorrelation}

Then, at the MS, the MS Cell Decision (MSCD) algorithm will be used to detect load values of BSs which are of interest to the MS and consequently make cell choice decision. We denote this MSCD algorithm using autocorrelation as MSCD_AutoCorr. The MS first correlates the preamble code with the copy of the original preamble sequence code list it possesses to identify the BSs (this step is involved in detecting the three strongest BS signals in lines (2 - 6) of MSCD_AutoCorr) and then uses the autocorrelation property (line 8 - 10) to find multiple peaks (if applicable) instead of the single peak which would have been the normal case. Detecting the number of peaks (line 11-13), it arrives at the value $j$ which conveys infor- 
mation about the load, which is then used to decide on the choice between the three BSs of interest.Prior to the Preamble Modifying Algorithm, the grouping intervals of the load values are assumed to have been decided by the central network controller using uniform grouping.

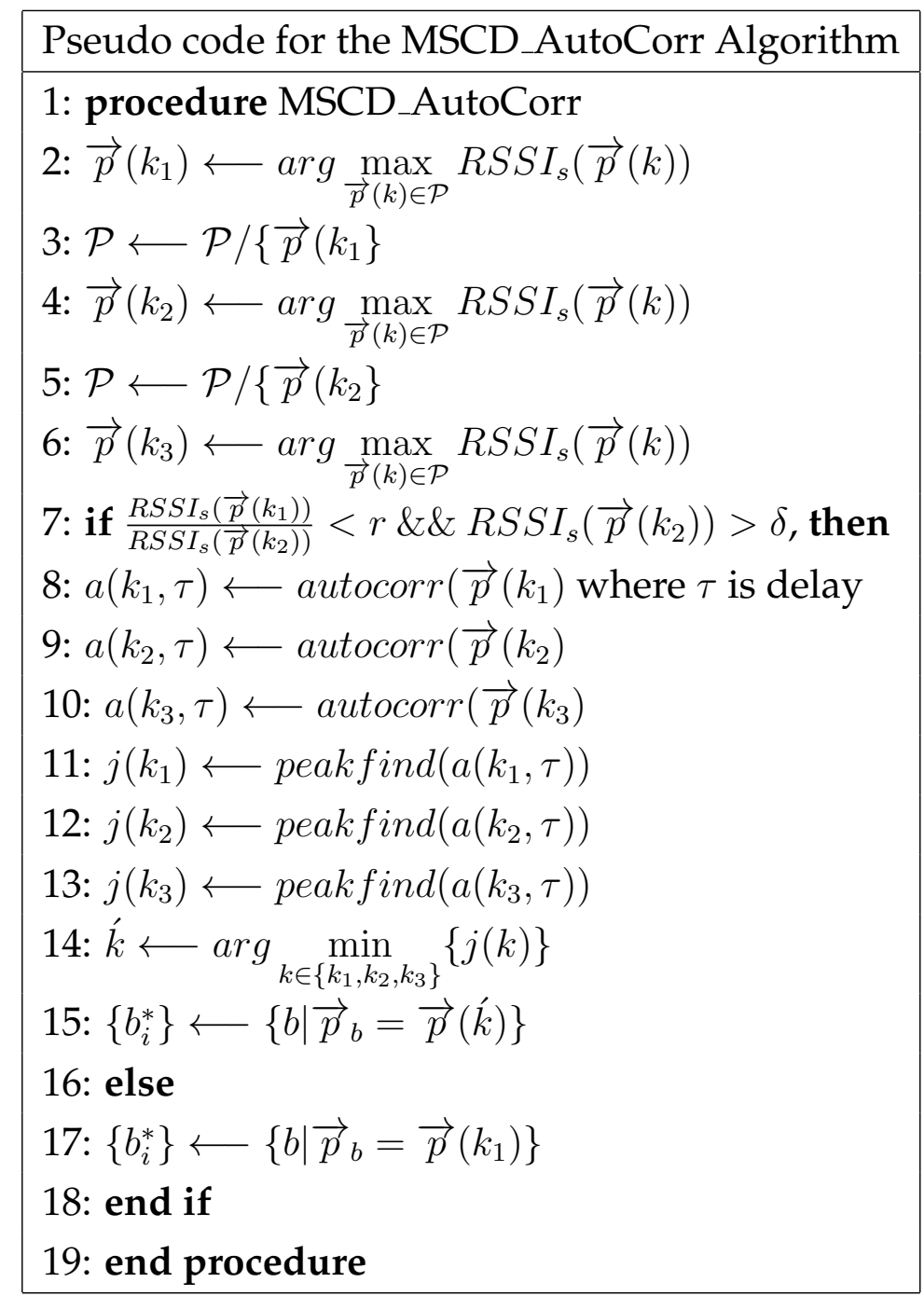

Table 4.2: MSCD Algorithm

The MSCD algorithm using autocorrelation (denoted as MSCD_Autocorr) is given as above. The peakfind() function finds the number of peaks in the argument plot for different values of $\tau$ and returns a value which is 
equal to the number of peaks in it. Here we define two design parameters $r$ and $\delta$, which specifies the area to be influenced by load balancing algorithm and the minimum RSSI requirement, respectively.

\subsection{Summary}

In this chapter, we first presented a brief defintion of the auto-correlation property of preamble sequences, which is the cross-correlation of a signal sequence with itself along with its use and applications. Based on this property, we have developed and defined two algorithms working side by side, one on the central network controller and one on the user side to associate MSs with BSs considering the level of load at them.

In the first algorithm, at the central network controller, the load values of all BSs is collected first and then the preamble sequences of all of them are modified. Then the controller assigns a specific value obtained by the modification to BSs according to their loads. The BSs will then transmit using these modified preamble sequences in the Preamble Modifying Algorithm. The MS then receives and correlates the preamble code with the copy of the original preamble it had and finds the modification which implicitly conveys the load level to it and it chooses the BS which is lightest loaded in the MSCD algorithm, which runs on the user's side.

In the next chapter, we present our proposed cross-correlation based implicit load indication scheme and how it is applied for load balancing. 
38CHAPTER 4. AUTO-CORRELATION BASED IMPLICIT LOAD INDICATION SCHEME 


\section{Chapter 5}

\section{Cross-Correlation based Implicit Load Indication Scheme}

\subsection{The Cross-Correlation Property}

Cross-correlation is defined as a measure of similarity of two waveforms as a function of a time-lag applied to one of them. This is also known as a sliding dot product or sliding inner-product. It is commonly used for searching a long-duration signal for a shorter, known feature. It also has applications in pattern recognition, single particle analysis, electron tomographic averaging, cryptanalysis, and neurophysiology.

Cross-correlation is similar in nature to the convolution of two functions. Whereas convolution involves reversing a signal, then shifting it and multiplying by another signal, correlation only involves shifting it and multiplying (no reversing) [41].

$$
(f \star g)(t)=\int_{-\infty}^{\infty} f^{*}(\tau) g(t+\tau) \mathrm{d} \tau
$$

where $f^{*}$ denotes the complex conjugate of $f$. 
In the case of dicrete functions, the cross-correlation is defined as :

$$
(f \star g)[n]=\sum_{m=-\infty}^{m=\infty} f^{*}[m] g(n+m)
$$

The cross-correlation is similar in nature to the convolution of two functions.
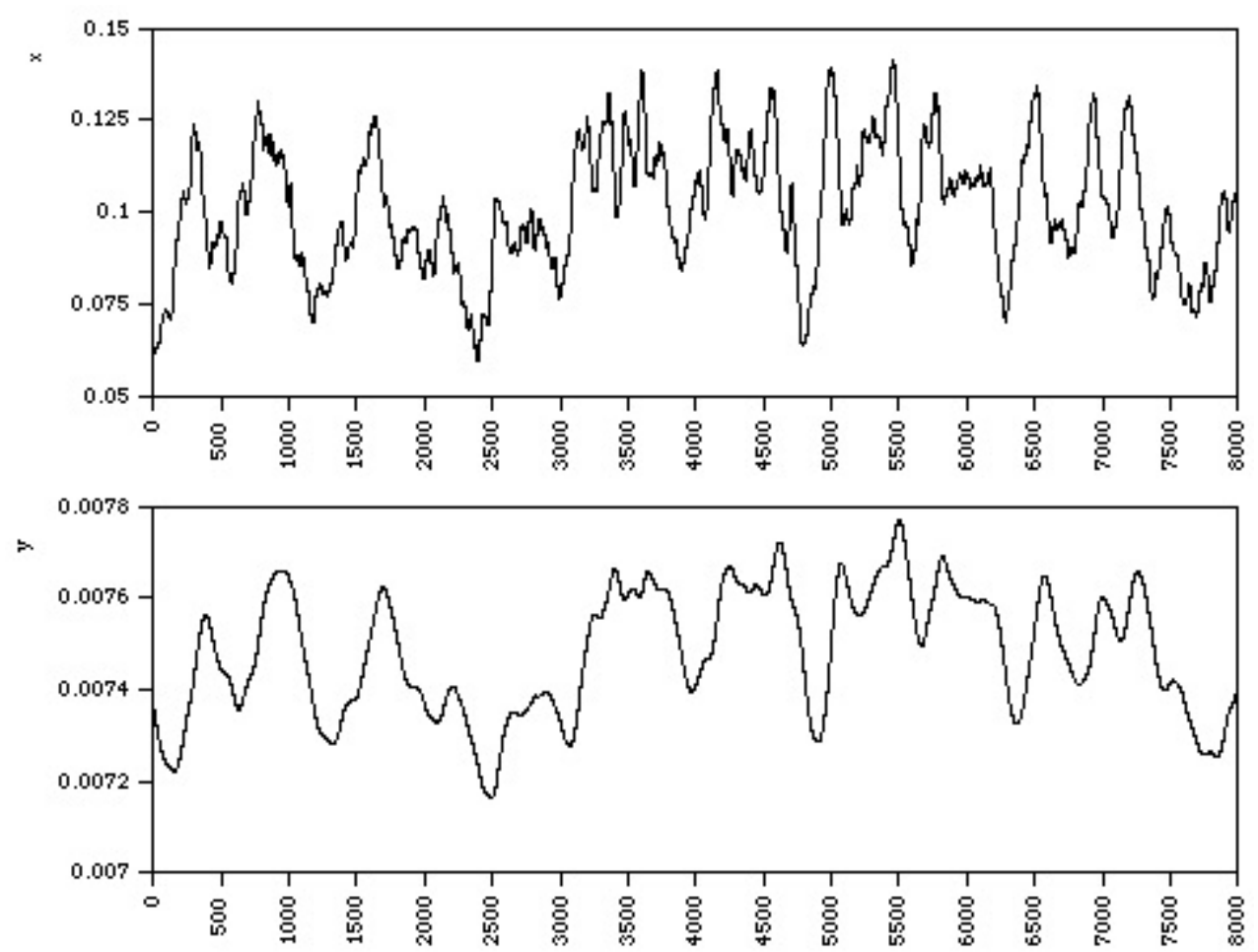

Figure 5.1: Two time series $\mathrm{x}, \mathrm{y}$.

As an example, Figure 5.1 shows two time series xy, and Figure 5.2, a cross-correlation between the two time series performed with a maximum delay of 4000. The strong correlation can be observed from the peak at the delay of about 40. 


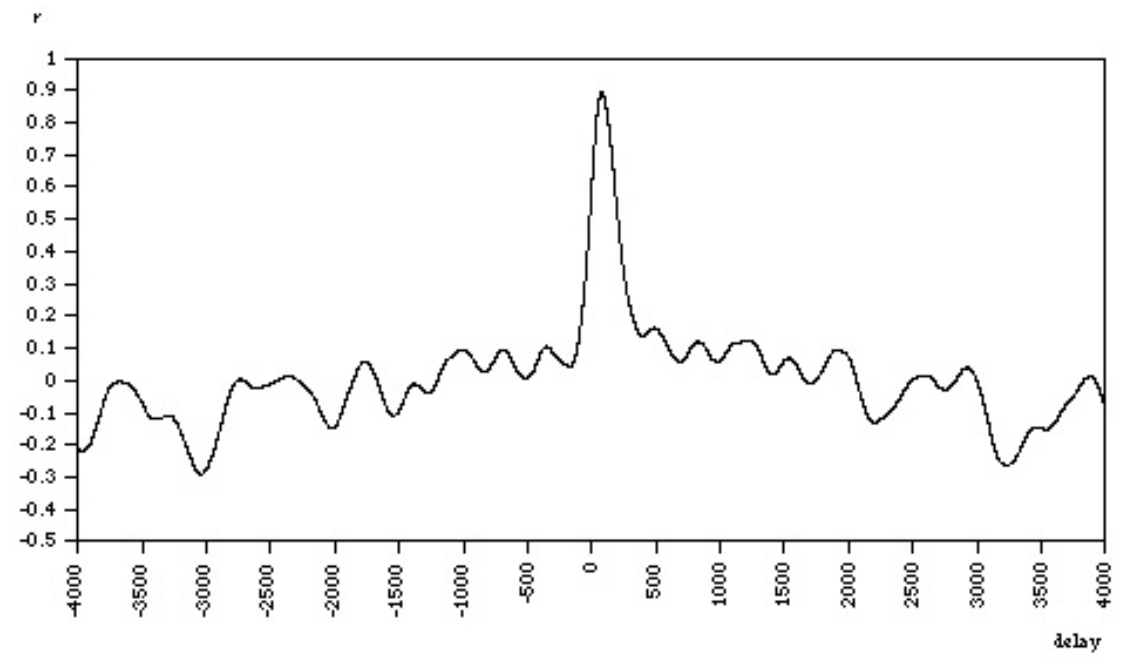

Figure 5.2: The cross-correlation series with a maximum delay of 4000 showing a strong correlation at a delay of about 40 .

\subsection{Preamble Allocation using Cross-correlation}

In this approach, what we call the Preamble Allocation using Cross-correlation $(P A C)$ algorithm is performed at the centralized controller. Here, we use the maximum correlation value of the preamble with a particular predefined sequence $\vec{p}_{0}$. This sequence can be an arbitrary preamble sequence taken from one of the $114 \mathrm{PN}$ codes which are used in the 1k FFT mode in IEEE 802.16e. When we correlate the BS's preamble code with this particular code $\vec{p}_{0}$, the result can then be used as a metric to which we can implicitly link the load of the BS. This is largely dependent on getting different such cross-correlation values for different preamble sequences. In this method, the set $\Phi_{s}$ contains the sorted list of various preamble sequences sorted with respect to their cross-correlation value with $\vec{p}_{0}$. In $\Phi_{s}$, the first preamble sequence has the lowest cross-correlation with $\vec{p}_{0}$ and the last one has the highest cross-correlation with $\vec{p}_{0}$.

Before the commencement of this algorithm, the central network controller will obtain the load values of all the base stations. This load could 
be the number of users connected or the number of calls connected. After this, the controller would create a list $\Phi_{s}$ which would contain the preamble sequences sorted according to the value of their maximum crosscorrelation with the reference preamble sequence $\vec{p}_{0}$. The algorithm then searches the most loaded BS in the set $\mathcal{B}$ (line 4). It then assigns the first sequence in $\Phi_{s}$, which is the one with the least correlation with $\vec{p}_{0}$ to it (line 5). Then, the pointer to the top of the list $\Phi_{s}$ is incremented to the sequence with the next lowest correlation with $\vec{p}_{0}$ and the whole process is repeated till all the BSs are exhausted.

\begin{tabular}{|l|}
\hline Pseudo-code for the PAC Algorithm \\
\hline 1: procedure $\operatorname{PAC}(\mathcal{B})$ \\
2: $i=1$ \\
3: while $\mathcal{B} \neq \emptyset$ do \\
4: $b \longleftarrow \arg \max _{b \in \mathcal{B}} l_{b}$ \\
5: $\overrightarrow{p_{b}} \longleftarrow \vec{p}(i)$ s.t. $\vec{p}(i) \in \Phi_{s}$ \\
6: $i \longleftarrow i+1$ \\
7: $\mathcal{B} \longleftarrow \mathcal{B} /\{\dot{b}\}$ \\
8: end while \\
9: end procedure
\end{tabular}

\subsection{BS Selection Algorithm using Implicit Indi- cation}

Together with the PAC algorithm, the MSCD using Cross-Correlation (denoted as MSCD_CrossCorr) algorithm is employed by the MS if it is near 
the boundary between three BSs. Firstly the algorithm identifies the three nearest BSs by using their RSSI (line 2 - 6). Then, a condition is tested using a design parameter $r$ which determines whether the MS is at the boundary area between the two identified BS's cells by calculating the ratio of their RSSI.

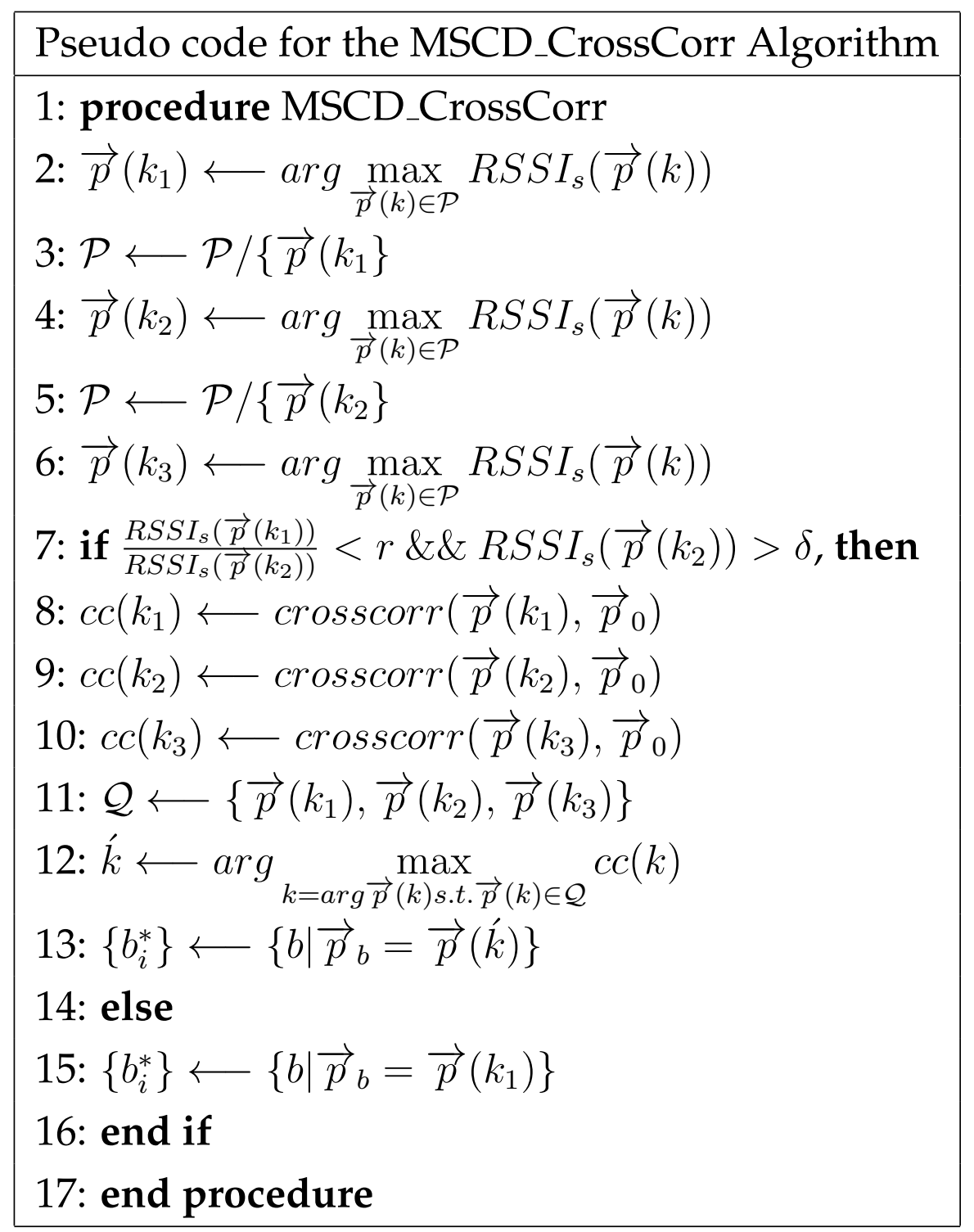

Also, a minimum RSSI criteria is tested using a parameter $\delta$ (line 7). When 
either of these two conditions fail, the usual RSSI based selection is followed (line 15). Otherwise, the load balancing algorithm comes into effect. Cross correlations of the preamble sequences of the three BSs are calculated (line 8 - 10) and the BS having highest maximum cross-correlation is chosen.

\subsection{Summary}

In this chapter, we first presented a brief defintion of the cross-correlation property of preamble sequences, along with its use and applications. We have developed and defined two algorithms working side by side on the basis of this property, one on the central network controller and one on the user side to associate MSs with BSs considering the level of load at them.

In the first algorithm that we call Preamble Allocation using crosscorrelation (PAC), at the central network side, the load value of all BSs is collected first and then the preamble sequences are correlated with an arbitary sequence to get different values which are then assigned to implicitly indicate the load to the users seeking association and service. On the user's side runs the MSCD algorithm, where the users correlate the sequences present in them with the sequence of the incoming stations to implictly know the load of the BS for smart cell-selection.

In the next chapter, we perform extensive simulations of our proposed schemes and discuss the results. 


\section{Chapter 6}

\section{Simulation Results and Discussion}

In this chapter, simulations results are presented on the two load balancing schemes described in Chapter 4 and 5. The main objective is to investigate the performance of the schemes to substantiate their effectiveness using popular performance metrics such as throughput, load variance and fair resource sharing. First, Section 6.1 discusses the channel characteristics in wireless systems that affect the performance and following that a breif note on the simulation environment. Subsequently, Section 6.2 presents the performance results using the performance metrics mentioned earlier. After this, in Section 6.3, the performance of our proposed schemes is then evaluated under different environments, namely, the Hata for urban areas. Finally, in Section 6.4, we present a comparison of our proposed schemes against RSSI-based schemes.

\subsection{Simulation Setup}

In this section, we first define the channel characteristics and emphasize how they affect the performance of a system and following it, the simulation environment. 


\subsubsection{Channel Characteristics}

In a typical outdoor wireless propogation environment, when a mobile wireless user communicates with a BS, the signal transmitted from the mobile user reaches the BS either directly (line-of-sight) or through multiple reflections on local scatterers such as buildings, mountains, etc. This is because the received signal strength is affected by multiple random attenuations and delays. Moreover, the mobility of either the mobile users or the scatterers may cause these random fluctuations to vary over time. Finally, in a shared wireless environment, the transmitted signal may come across interference due to concurrent transmissions.

The attenuation incurred in wireless propogation can be classified into three main elements : a signal attenuation due to the distance between communicating nodes (path-loss), attenuation effects due to absorption in local structures such as buildings (shadowing), and rapid signal fluctuations due to constructive and destructive interfeerence of multiple reflected radio wave paths (fading).

Variations due to path-loss and shadowing occur over relatively large distances that happens as mobile users move through a distance of the order of the cell. Path-loss and shadowing are typically frequency dependent. Variation due to multi-path fading occurs over very short distances, on the order of the signal wavelength and is frequency dependent. The channel characteristics depend on the combination of all three propogations effects mentioned, but for simplicity we only consider a noisy channel with path-loss attenuation for our simulations.

\subsubsection{Simulation Environment}

We consider a 12-cell model with a frequency reuse factor of 1 , as illustrated in Figure 6.1, and focus on the three cells in the center with numbers 0,1 and 2 highlighted.

For the initial distribution of users, we assume that 100 stations are lo- 


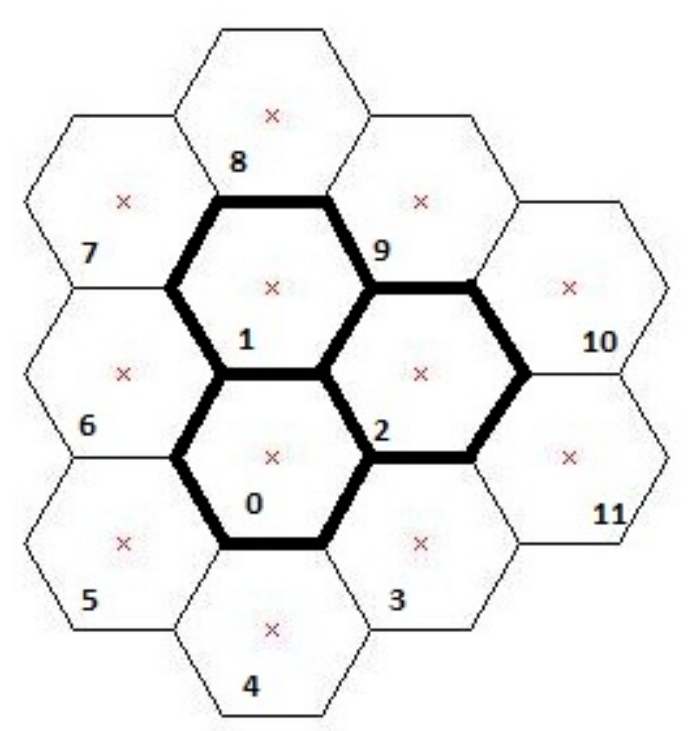

Figure 6.1: A conventional 12-cell model with focus on cells 0,1 and 2

cated in cell 0,80 in cell 1, 20 in cell 2, and all the other cells have 45 stations randomly distributed over their cell region. Our algorithm is then applied to the boundary stations. In our simulations, we consider the channel gain due to path loss effect with a path loss exponent of 4 , which is ideal for a typical cellular network.

\subsection{Simulation Results and Discussion}

In this section, simulation results are presented along with the underlying assumptions. The simulations implement the proposed algorithms at the decision making level where depending on the load of BSs, the stations in a certain area (defined by $r$ ) near the boundary choose the BS to be associated with. Hence, as both algorithms look at the same quantity i.e. load to help MS's make the cell choice, the simulation results are identical. 


\begin{tabular}{|l|l|}
\hline Channel Prameters & Value \\
\hline Bandwidth $(\mathrm{MHz})$ & 10 \\
\hline Transmission Power $(\mathrm{db})$ & 20 \\
\hline Path Loss Exponent & 4 \\
\hline
\end{tabular}

Table 6.1: Channel parameters for simulation

The differences in the two algorithms lie in the method of detection of load information in the preamble, and thus when this is accurately done as assumed in the simulations, both algorithms give identical results. Figure 6.2 shows that the number of users becomes more evenly distributed as $r$ increases. This is where the red MSs are associated to heavily loaded cell 0 , blue MSs are associated to cell 2 with heavy load again and the green MSs are associated with the lightly loaded cell 1.

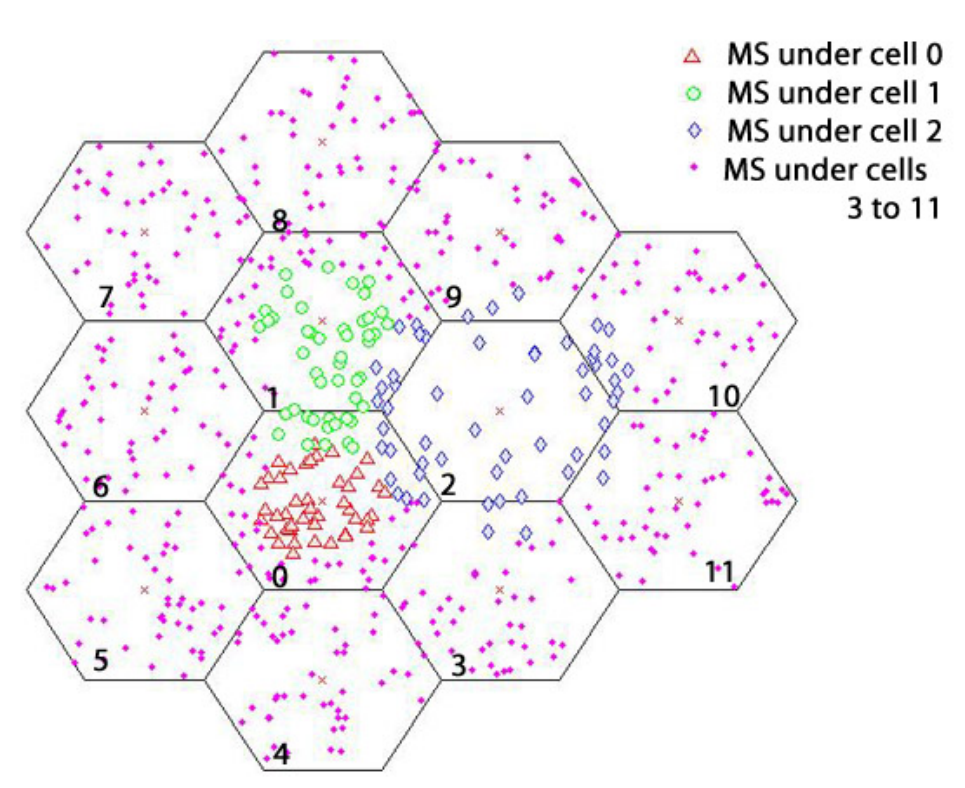

Figure 6.2: Distribution of users in cells at $r=10$

At $r=10$ case, one can see how our algorithm leads many of the MSs near the boundary of cell 0 to be handed over to neighbouring BSs and thus 
becoming green/blue/yellow, as cell 0 is heavily loaded. Similarly, blue MSs are also handed over to cells depending on their loads. Green MSs, however, are mostly those who have been handed over to BS of cell 1 by other heavily loaded BSs.

\subsubsection{Load Variance Test}

As a quantitative measure of how the load is distributed among the various BS's cells, we propose a metric called the Load Variance $l_{\text {var }}$ which is given by:

$$
l_{\text {var }}=\frac{\sum_{b \in \mathcal{B}}\left(l_{b}-\bar{l}_{b}\right)^{2}}{n(\mathcal{B})}
$$

where $n(\mathcal{B})$ is the total number of BSs, $l_{b}$ is the load experienced by each BS and $\bar{l}_{b}$ is the mean of all the load values.

For this test, we take the number of users as 100 in cell 0, 80 in cell 1, and 20 in cell 3 . Therefore, as the load gets distributed more evenly among the different BSs, we see a gradual decrease in $l_{v a r}$, as seen in Figure 6.3, which shows its variation with different values of our design parameter $r$.

We now vary the number of user in cells and simulate by having 60 users in cell 0,40 users in cell 1 , and 10 users in cell 2. We observe, at $r=$ 7, in Figure 6.4, the load gets minimal and then slightly increases before stabilizing after $r=9$. This is because the total population decreases a bit as $r$ increases in cell 2 and go into cell 10 and 11 as their serving cells. Thus, this metric is useful in measuring the even-ness of distribution of MSs in a collection of cells and shows the effectiveness of our proposed schemes in terms of fair user distribution. 


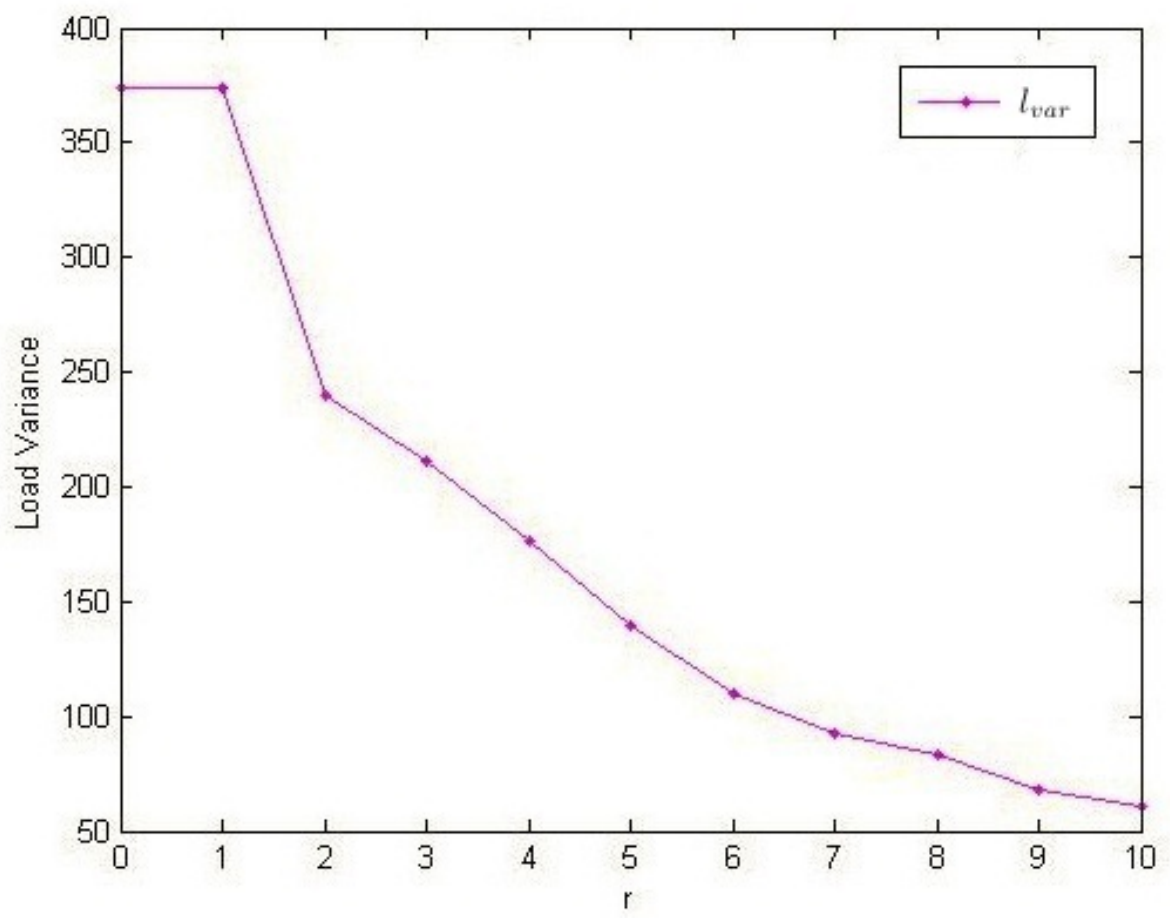

Figure 6.3: Load Variance test with 100 users in cell 0, 80 users in cell 1, and 20 users in cell 3.

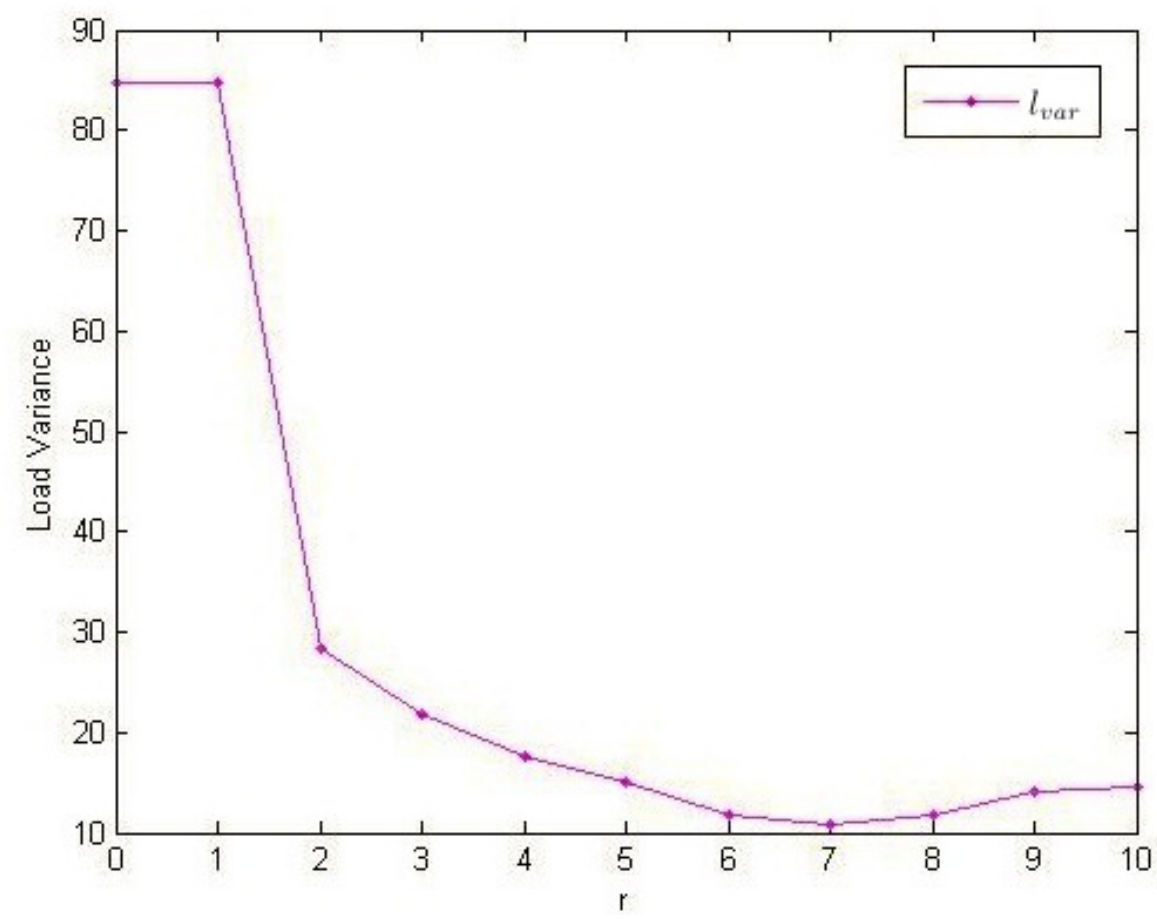

Figure 6.4: Load Variance test with 60 users in cell 0, 40 users in cell 1, and 10 users in cell 2. 


\subsubsection{Resource Fairness Test}

We evaluate the effect of load balancing over multi-cells in terms of the corresponding fairness criteria when the resource allocation in every cell follows resource fair (RF) scheduling policy. Since the resource allocated to each user in each single cell is inversely proportional to the number of users in that cell, it is good load balancing from the viewpoint of RF scheduling, resulting in users in every cell being distributed as evenly as possible.

As we see in Figure 6.5, at $r=10$, all three BSs of cells 0 to 2 have number of associated MSs between 50 to 60 and initially the number of users were 100 in cell 0, 80 in cell 1, and 20 in cell 2 .

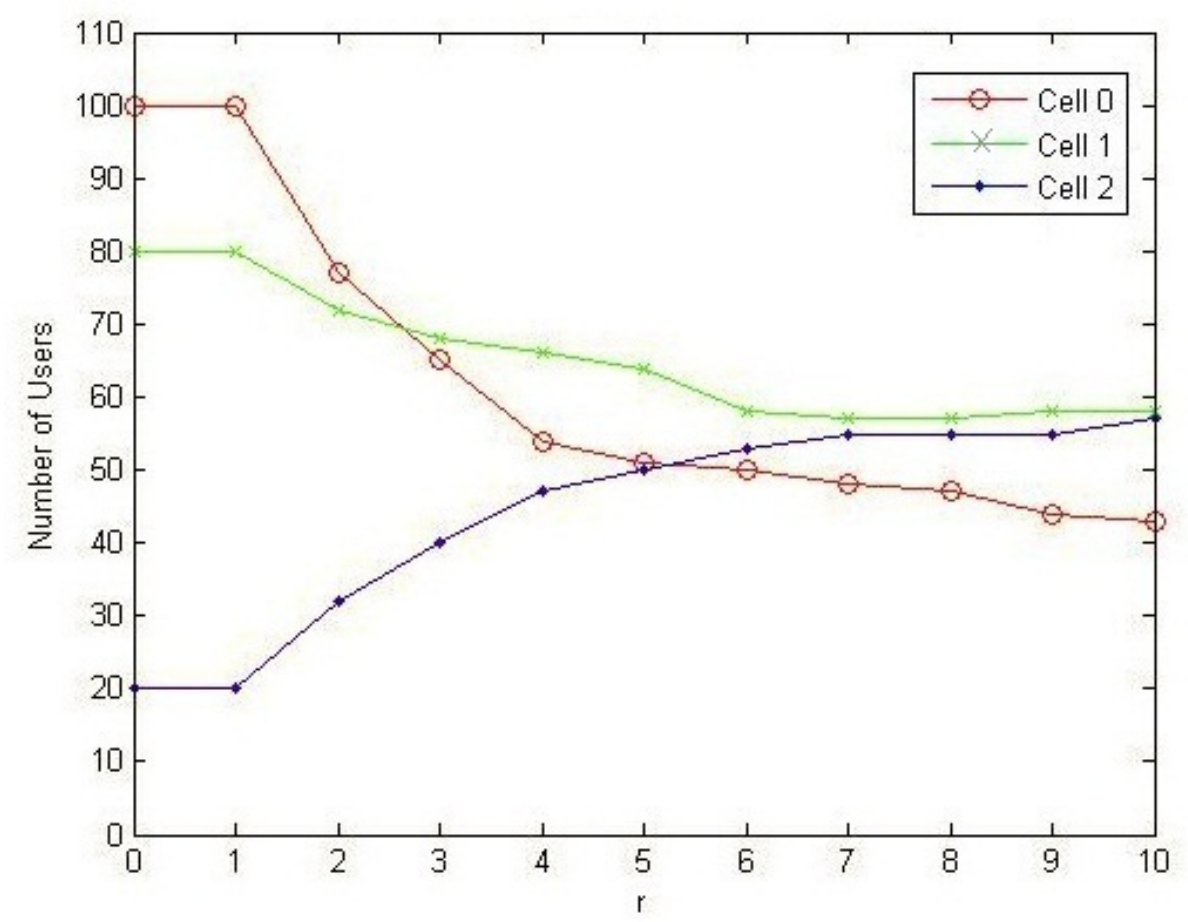

Figure 6.5: Resource Fairness with initially 100 users in cell 0, 80 in cell 1 and 20 users in cell 2 . 
We now vary the number of user in cells and simulate by having 50 users in cell 0, 40 users in cell 1, and 10 users in cell 2 in Figure 6.6. We again observe that the number of users get even in cells 0 to 2 . This can be interpreted that our load balancing scheme leads to an improved consequence in terms of fair resource sharing.

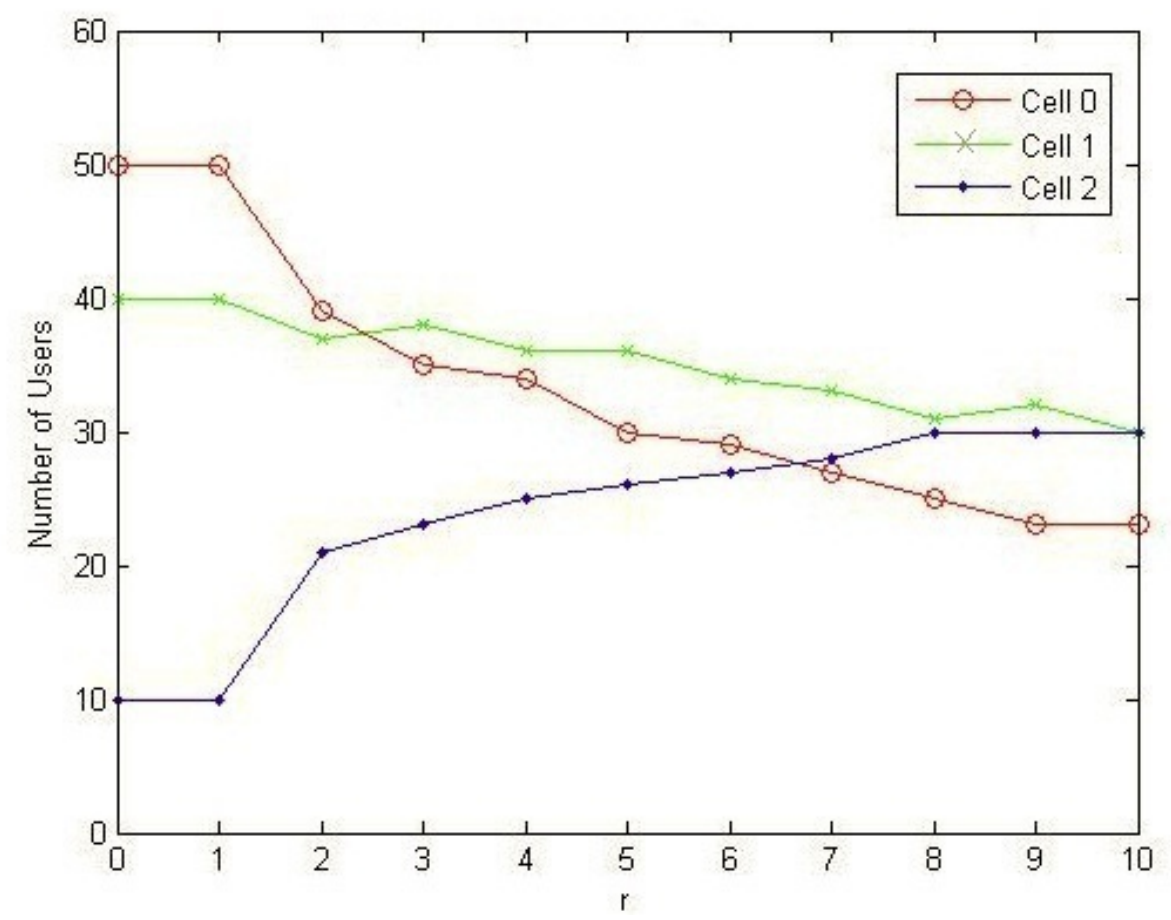

Figure 6.6: Resource Fairness with initially 50 users in cell 0, 40 in cell 1 and 10 users in cell 2.

\subsubsection{Spectral Efficiency Test}

For minimum throughput simulations, we consider the lower bound case for throughput in a cell that occurs when all the users are at the maximum distance from the BS, and hence, have the lowest possible SNR (Signal to Noise Ratio) at the MS. In such cases, the bandwidth is equally distributed among the MSs, but the capacity reaches its minima because of lowest 
SNR. This is clear from the expression for channel capacity [34], where $R$ is the channel capacity, $W$ is the bandwidth assigned to each MS, $P$ is the power received by the MS and $N_{0} / 2$ is the noise.

$$
\frac{R}{W}=\log \left(1+\frac{P}{N_{0} W}\right)
$$

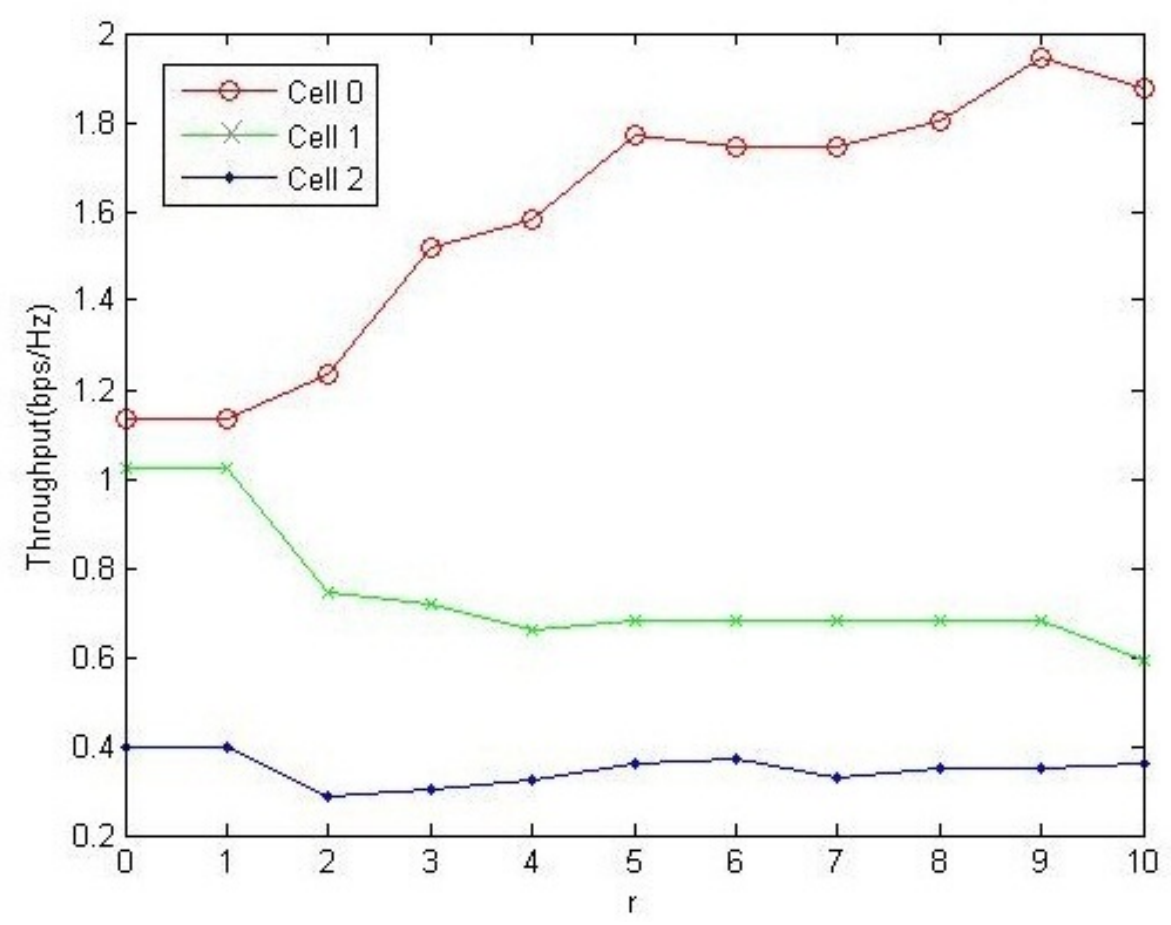

Figure 6.7: Throughput Fairness with initially 100 users in cell 0, 80 in cell 1 and 20 users in cell 2.

Thus, this lower bound of throughput in a cell is calculated for different variations of $r$ and it is seen that the minimum throughput improves considerably for the heavily loaded BS on application of the proposed algorithms for load balancing. This is because the initially heavily-loaded BS hands over some of its boundary users to the adjoining cells and hence, achieves better throughput. 
On the other hand, the other two lightly loaded BS's throughput deteriorates slightly because of the increase in number of users managed by the BS, as seen in Figure 6.7.

We now vary the number of user in cells and simulate by having 50 users in cell 0,40 users in cell 1, and 10 users in cell 2 in Figure 6.8. We again observe that the minimum throughput of users in cell 0 increases along with users in cell 2, while the minimum throughput of users in cell 1 experience a deterioration in their throughput due to the new handoffs.

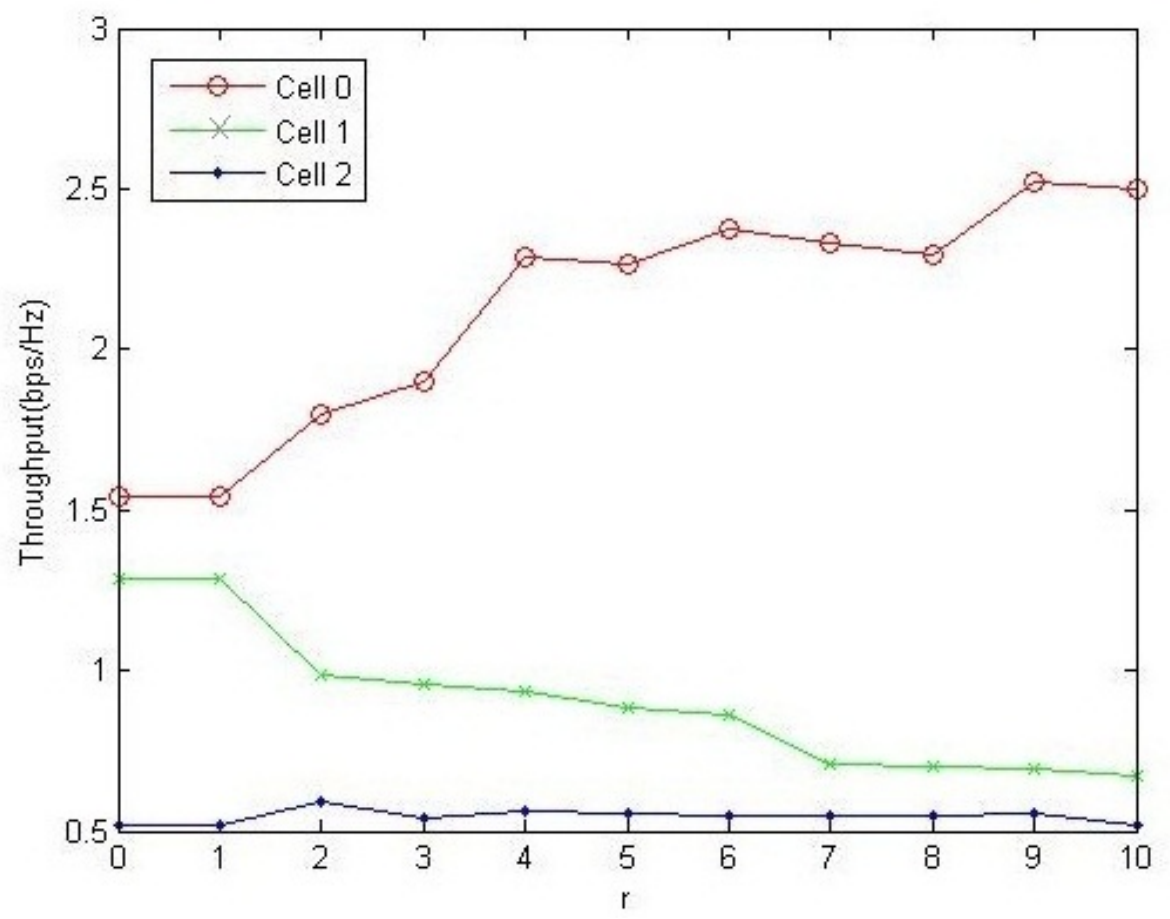

Figure 6.8: Throughput Fairness with initially 50 users in cell 0, 40 in cell 1 and 10 users in cell 2 .

This can be interpreted that our load balancing scheme leads to an improved consequence in terms of max-min throughput fairness. 


\subsubsection{Evaluation using Jain's Fairness Index}

The most simple way to evaluate how load balanced the system is, is to calculate the average load of the whole system:

$$
L=\frac{\sum_{i=1}^{n} U_{i}}{n}
$$

where $n$ is the number of BSs, and $U_{i}$ is the resource utilization of BS $i$ and compare this average to the individual resource utilizations $U_{i}$ of each BS. To describe the loading state of the whole system with one value the following load balance index has been defined [42]:

$$
B=\frac{\left(\sum_{i=1}^{n} U_{i}\right)^{2}}{n * \sum_{i=1}^{n} U_{i}^{2}}
$$

Figure 6.9 shows the load balance index, where values near 1 depict a load balanced system and 0 , no load balancing.

To quantify and measure the fairness acheived in the system, we use Jains's Fairness Index again described as follows :

$$
J\left(x_{1}, x_{2}, \ldots, x_{n}\right)=\frac{\left(\sum_{i=1}^{n} x_{i}\right)^{2}}{n * \sum_{i=1}^{n} x_{i}{ }^{2}} \quad[42]
$$

Equation 6.4 rates the fairness of a set of values where there are $n$ users and $x_{i}$ is the throughput for the $i$ th connection. The result ranges from $1 / n$ (worst case) to 1 (best case), and it is maximum whenall users receive the same allocation. This index is $k / n$ when $k$ users equally share the resource, and the other $n$ - $k$ users receive zero allocation. This metric identifies underutilized channels and is not unduly sensitive to atypical network flow patterns.

Figure 6.10 shows, as $r$ increases, the system gets fairer and at $r=6$, we are able to obtain fairness greater than 0.9 on the index. This also proves that there is a very strong correlation between load balancing and fairness. 


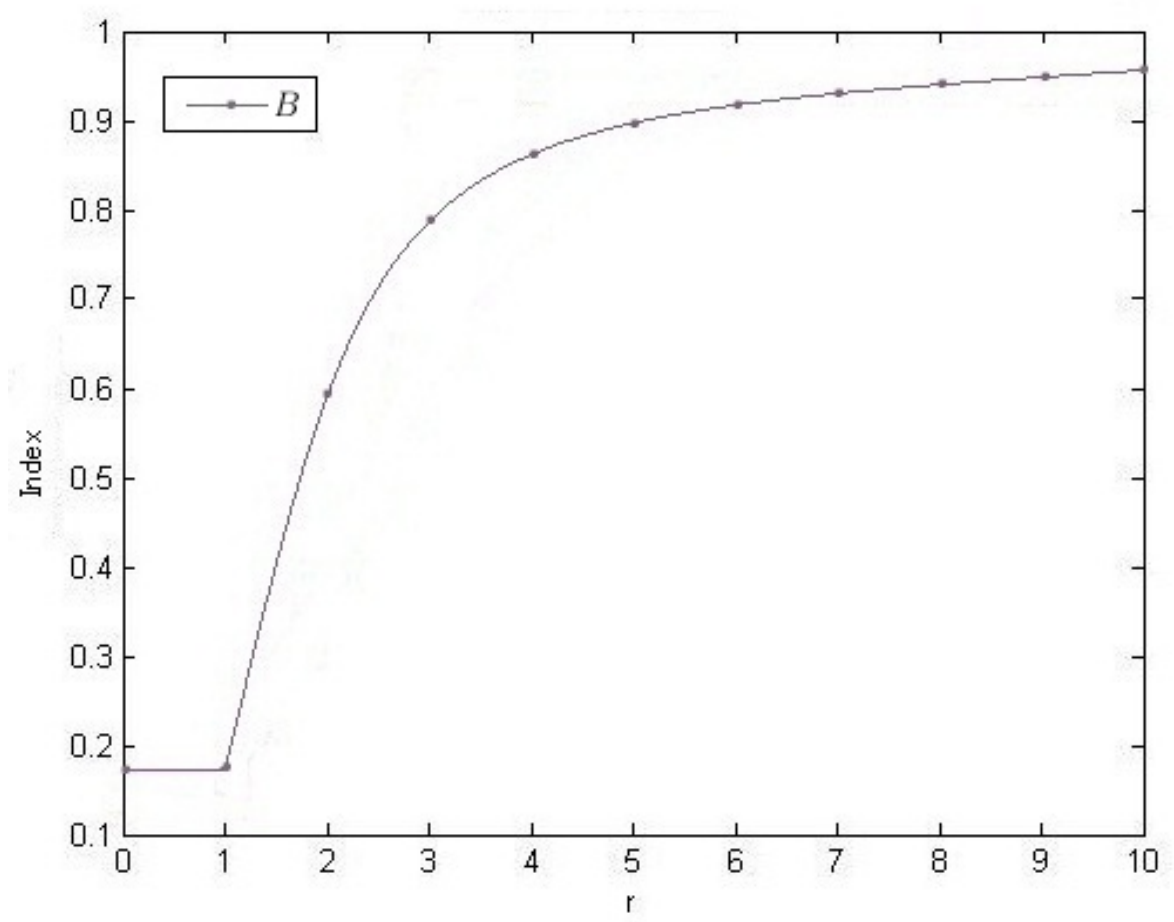

Figure 6.9: Load Balancing Index vs $r$

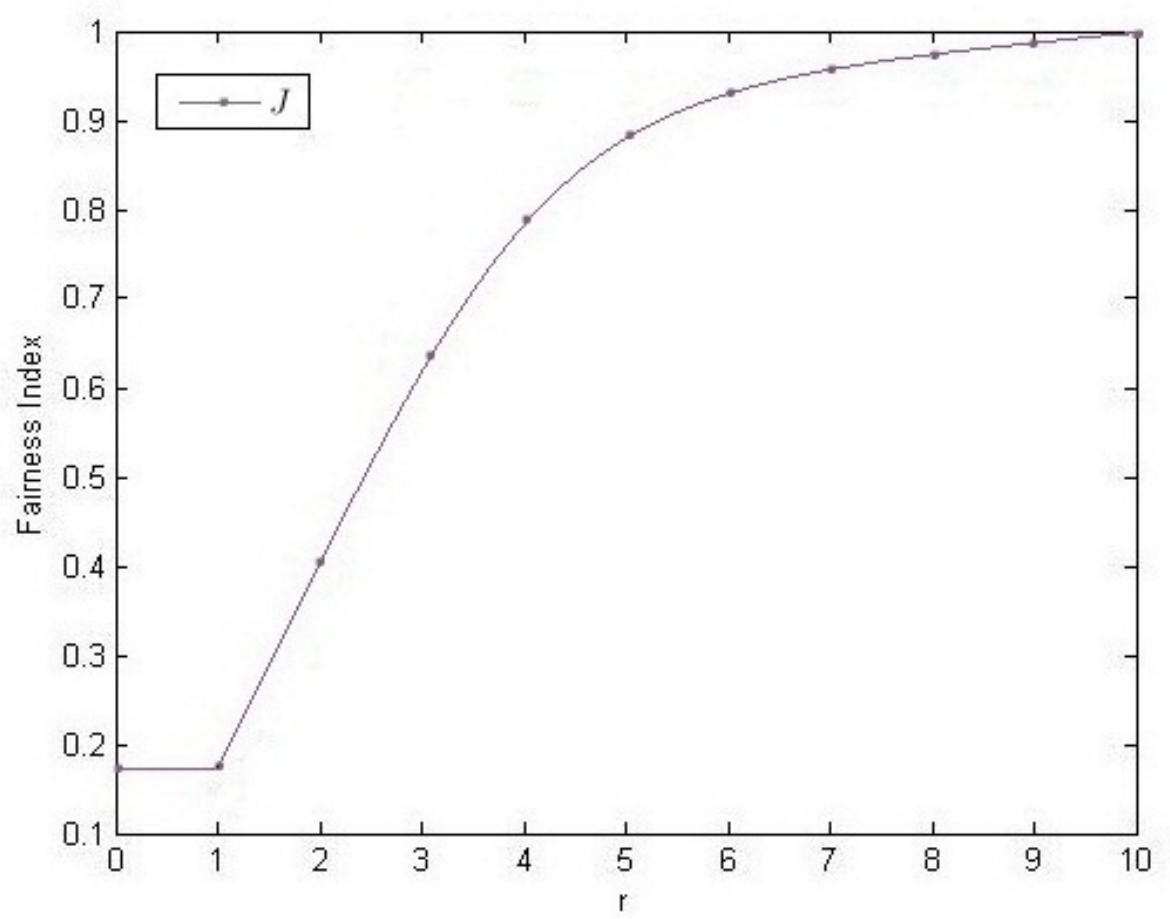

Figure 6.10: Jain's Fairness Index vs $r$ 


\subsection{Performance under Different Environments}

In wireless communication, the Hata Model for Urban Areas, also known as the Okumura-Hata model for being a developed version of the Okumura Model, is the most widely used radio frequency propagation model for predicting the behaviour of cellular transmissions in built up areas. This model incorporates the graphical information from Okumura model and develops it further to realize the effects of diffraction, reflection and scattering caused by city structures. We consider this model to evaluate the robustness of our schemes in different environments. Below mentioned are the coverage parameters considered which are a standard for the Hata model.

\begin{tabular}{|l|l|}
\hline Coverage & Value \\
\hline Frequency $(\mathrm{MHz})$ & $150-1500$ \\
\hline Mobile Station Antenna Height (Meters) & $1-10$ \\
\hline Base station Antenna Height (Meters) & $30-200$ \\
\hline Link distance (Kms) & $1-20$ \\
\hline
\end{tabular}

Table 6.2: Standard coverage parameters for the Hata model

The Hata model for Urban Areas [49] is formulated as :

$L_{U}=69.55+26.16 \log f-13.82 \log h_{B}-C_{H}+\left[44.9-6.55 \log h_{B}\right] \log d(6.6)$

where,

$L_{U}=$ Path Loss in Urban Areas. Unit: decibel (dB)

$h_{B}=$ Height of Base Station Antenna. Unit: meter $(\mathrm{m})$

$h_{M}=$ Height of Mobile Station Antenna. Unit: meter (m)

$f=$ Frequency of Transmission. Unit: megahertz $(\mathrm{MHz})$

$C_{H}=$ Antenna Height Correction Factor 
$d=$ Distance between the Base and Mobile Stations. Unit: kilometers $(\mathrm{km})$

In the next section, we will evaluate our schemes under the small and large city models to test how they perform under varying levels of path loss.

\subsubsection{Hata Model for a Small City}

We now simulate our schemes under the Hata - small city model with the equation for path loss being the same as above, but the value of the antenna height correction factor being different which is calculated as follows [49]:

$$
C_{H}=0.8+(1.1 \log f-0.7) h_{M}-1.56 \log f
$$

We first simulate for throughput fairness versus our design parameter $r$. Figure 6.11 shows the fairness of throughput under the channel conditions mentioned above. It can be observed that there is an improvement in the throughput of the cell 0 as the cell edge users leave the cell and are handed off to cell 1 and 2. Similarly, the throughput of cell 1 deteriorates as it accomodates new users from cell 0 and gets stabilized as $r$ increases. Due to the very less number of users in cell 2 , the throughput improvement is visible, but is minimal and stabilizes along with increasing $r$.

This shows that our proposed schemes fair well in terms of throughput fair scheduling, even though the improvement in the minimum throughput in not very substantial.

As seen in Figure 6.12, our schemes perform well to induce user handoffs into the lightest loaded cell, which is cell 2 and gains users from both cells 0 and 1 showing a well balanced user distribution under these channel conditions. This is mostly due to the effectiveness of our design parameter $r$ that targets the boundary users that the schemes are able to balance user distribution well. 


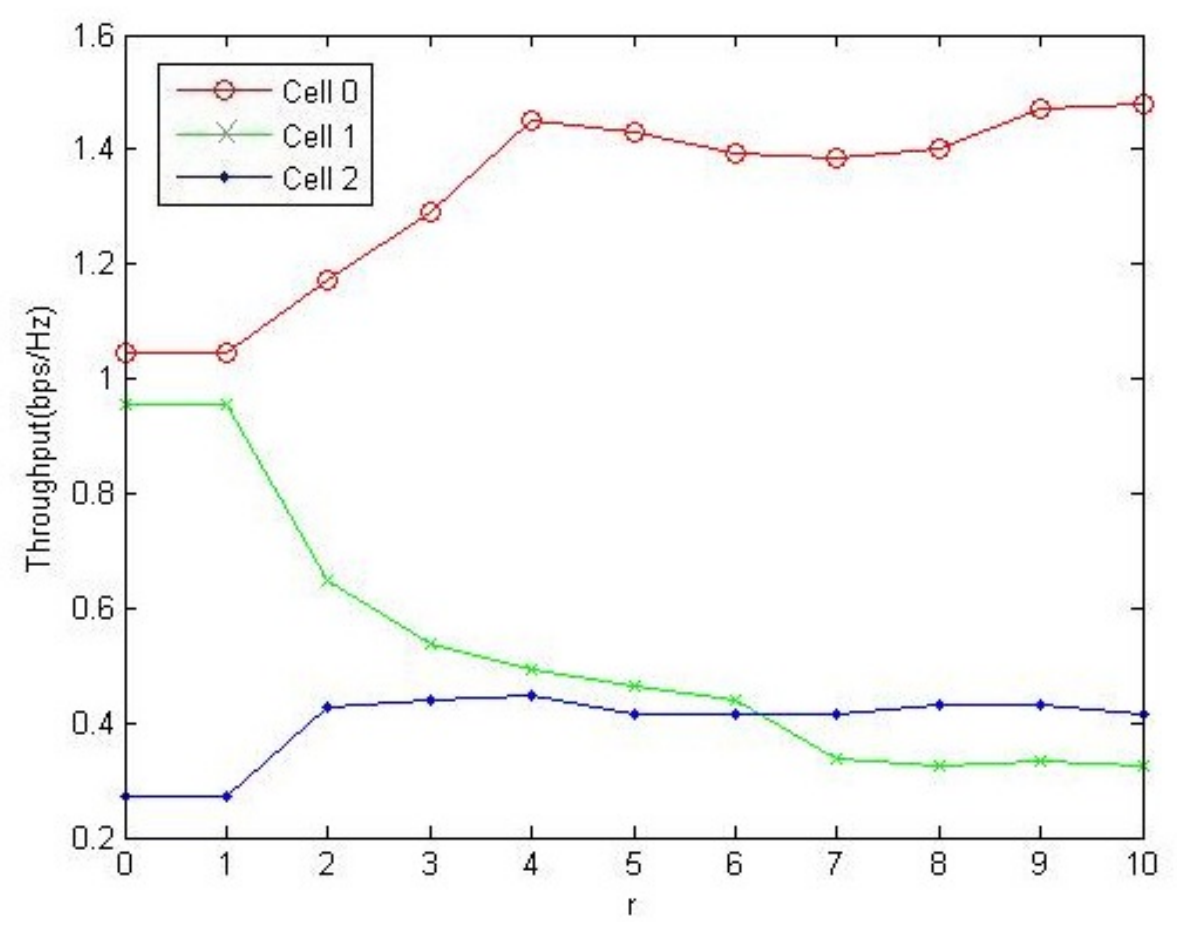

Figure 6.11: Throughout Fair vs $r$ for a small city

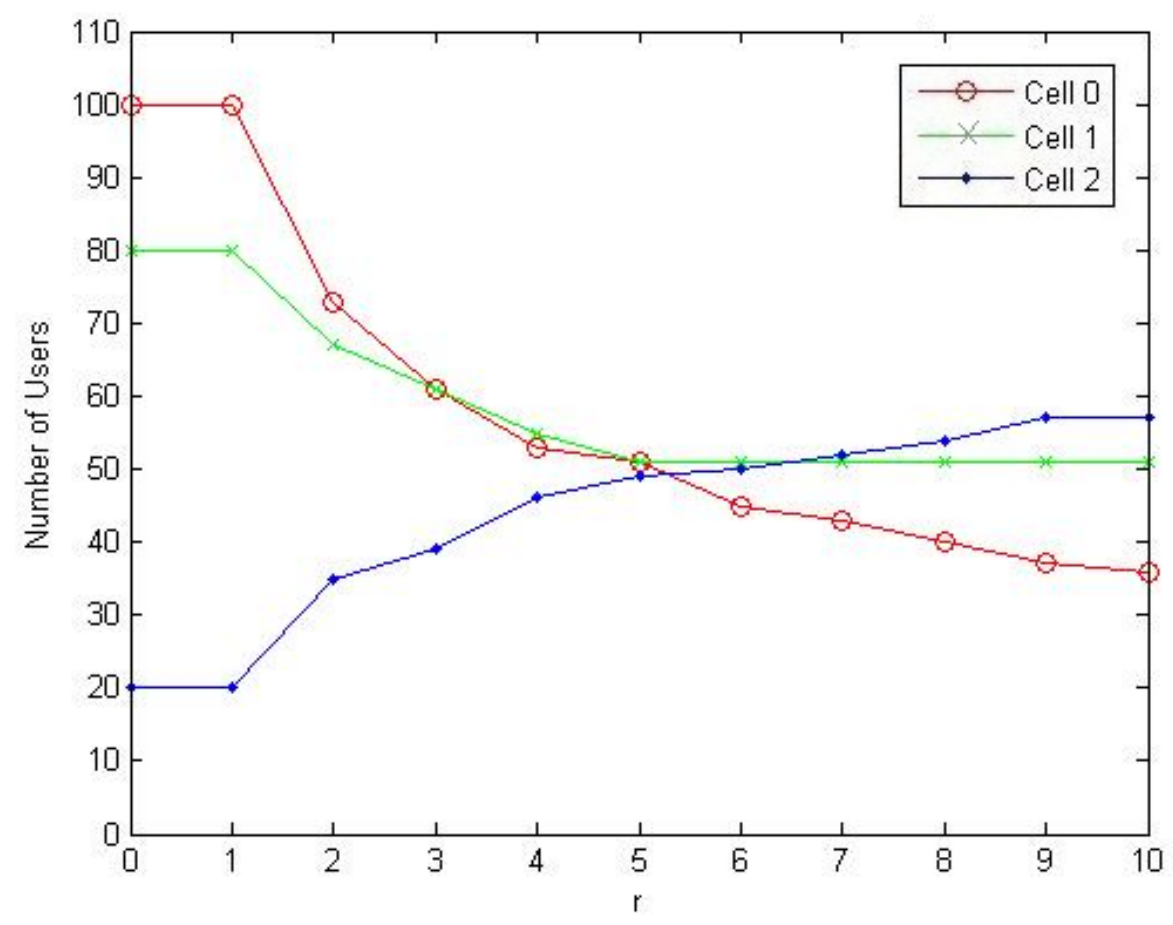

Figure 6.12: Resource Fair vs $r$ for a small city 


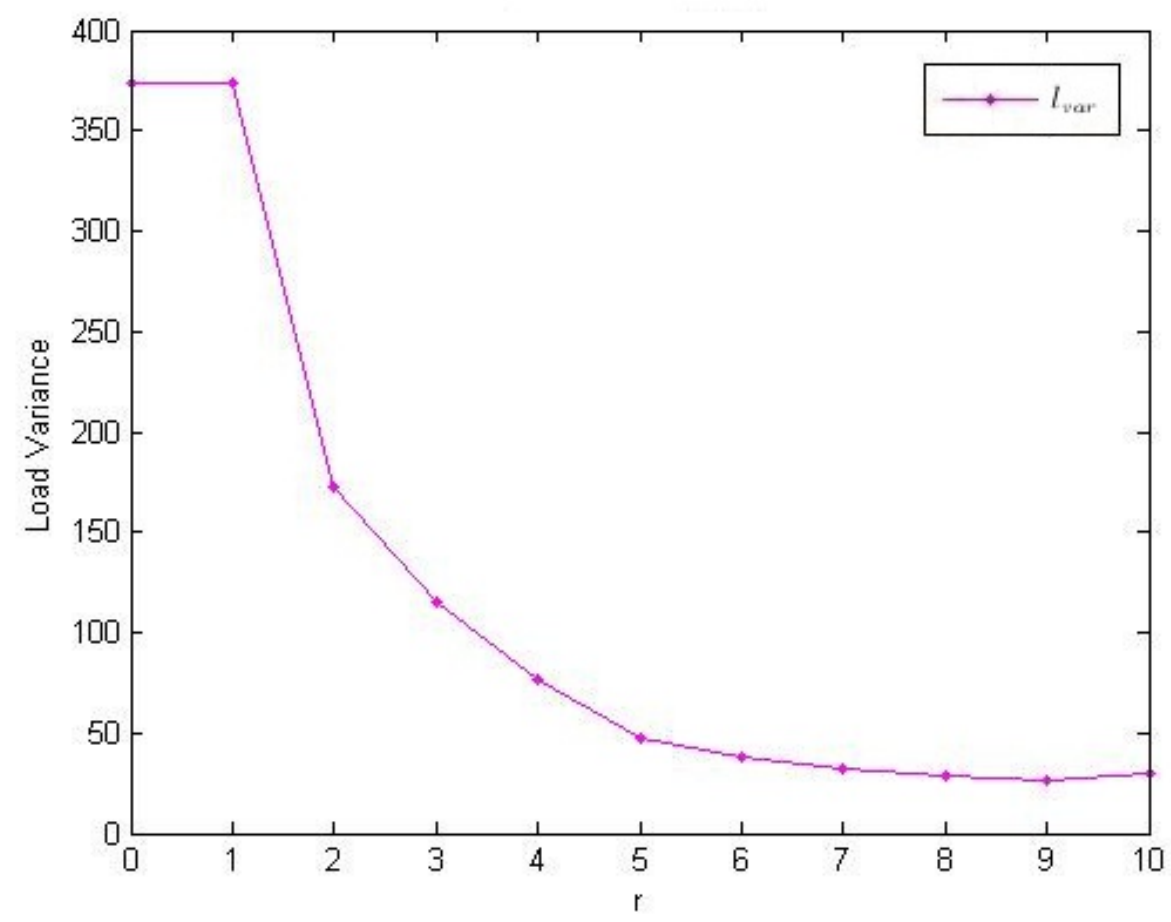

Figure 6.13: Load Variance vs $r$ for a small city

Figure 6.13 shows the varinace of load in the system. Due to the severity of noisy conditions, when our load balancing algorithm comes into effect at $r=1$, we see a sharp decline in the load variance initially until $r=2$ because of the user handoffs. Then the variance gradually stabilizes at $r=8$.

We now evaluate our schemes in terms of the load balance index of the whole system [42] now. It can be observed from Figure 6.14 that due to the noisy channel conditions, at $r=2$, when our load balancing scheme comes into effect, we see an increment initially but it is met by resistance from $r=3$ to 8 , until it sharply increases again at $r=9$. This shows the robustness of our schemes. 


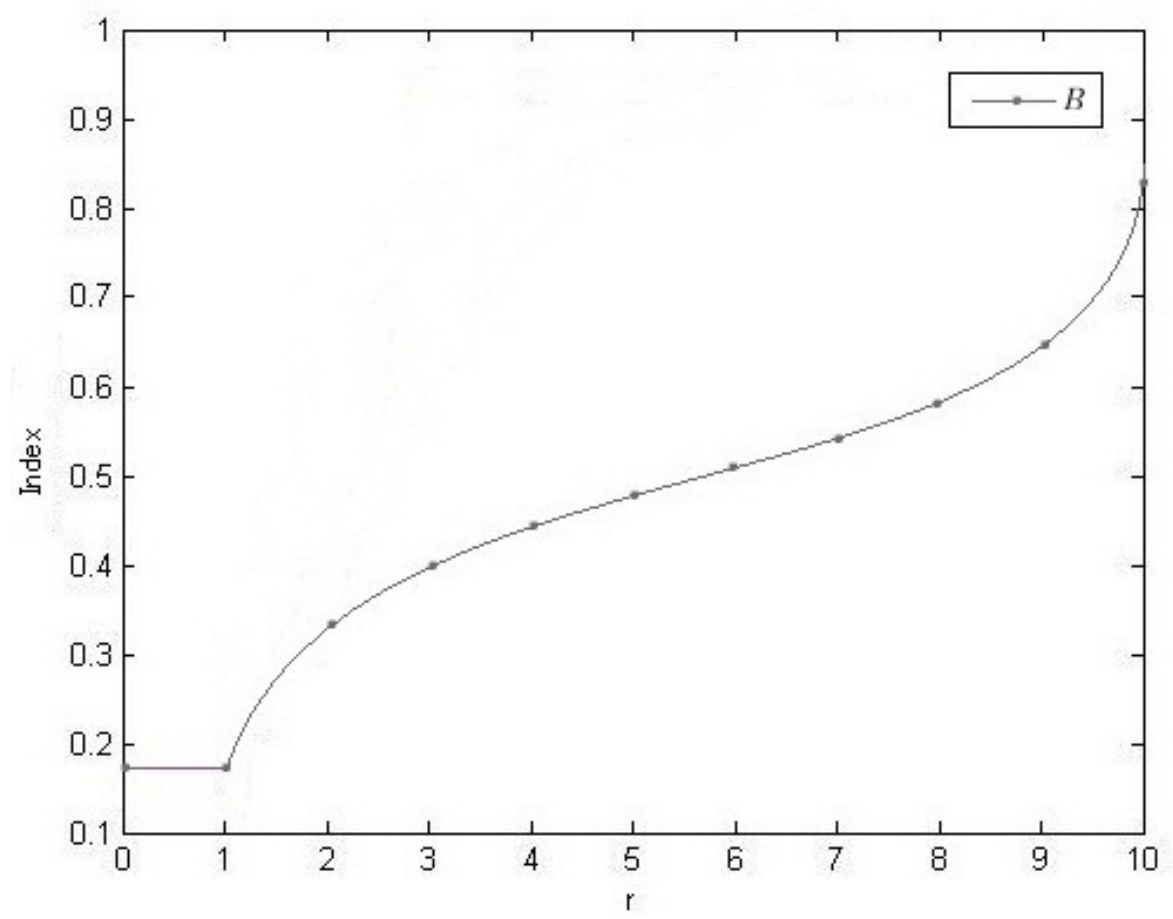

Figure 6.14: Load Balance Index vs $r$ for a small city

\subsubsection{Hata Model for a Large City}

We now simulate our schemes under the Hata - large city model with the equation for path loss being the same as above, but the value of the antenna height correction factor being different which is calculated as follows:

$$
C_{H}= \begin{cases}8.29\left(\log \left(1.54 h_{M}\right)\right)^{2}-1.1, & 150 \leq f \leq 200 \\ 3.2\left(\log \left(11.75 h_{M}\right)\right)^{2}-4.97, & 200<f \leq 1500\end{cases}
$$

We first simulate again for throughput fairness versus our design parameter $r$. Figure 6.15 shows the fairness of throughput under the channel conditions mentioned above. It can be observed that there is a great improvement in the throughput of the cell 0 as the cell edge users leave the 
cell and are handed off to cell 1 and 2 until $r=9$ where noisy conditions overpower it, but it recovers again at $r=10$.

The throughput of cell 1 deteriorates as it accomodates new users from cell 0 and gets stabilized as $r$ increases, but due to the extremely noisy channel conditions, it does not gain at all to be noticable. Also, due to the very low number of users in cell 2 , the throughput deterioration is barely visible, but is minimal and stabilizes along with increasing $r$.

This shows that our proposed schemes are able to perform in the noisiest of environments in terms of throughput fair scheduling, even though the improvement in the minimum throughput in not very substantial.

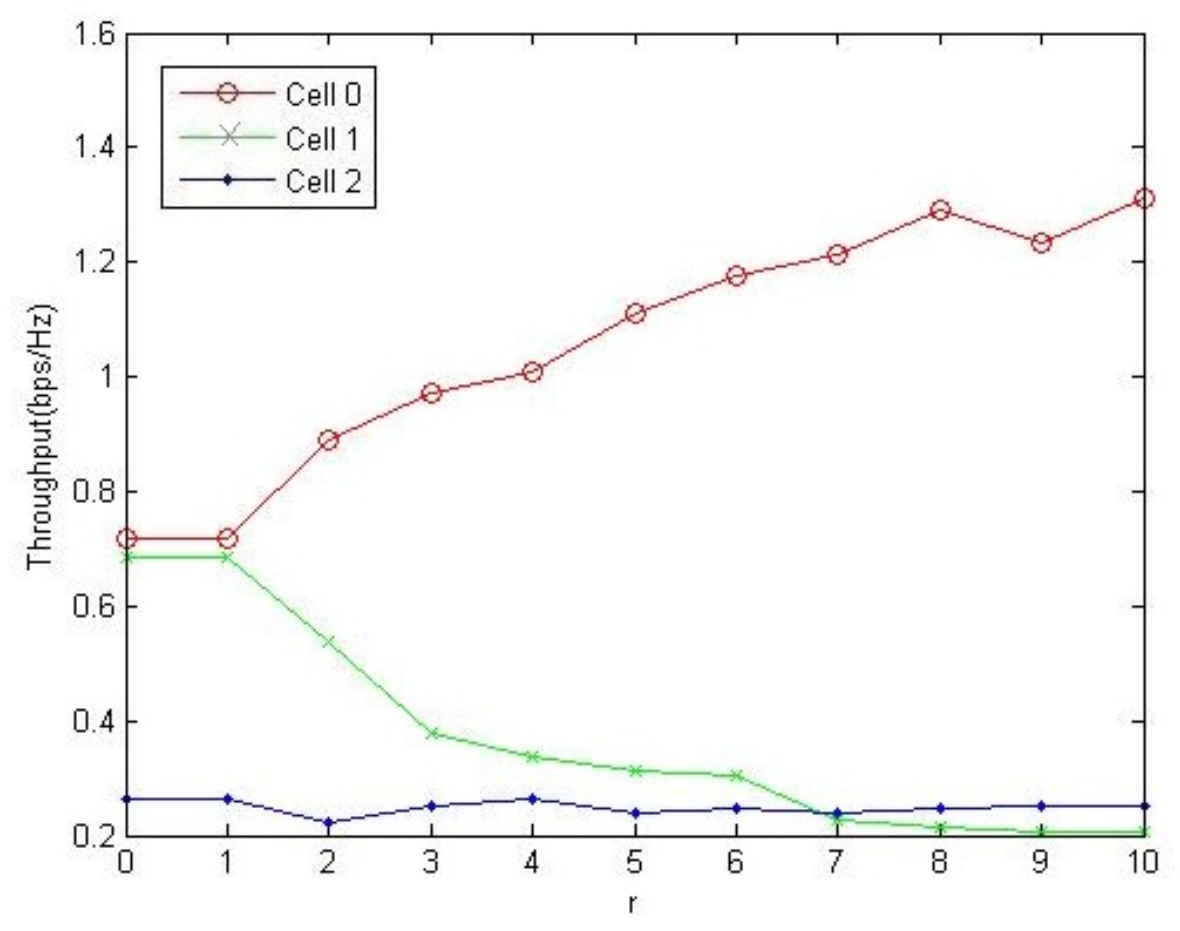

Figure 6.15: Throughout Fair vs $r$ for a large city

As seen in Figure 6.16, our schemes perform well again to induce user handoffs into the lightest loaded cell, which is cell 2 and gains users from 


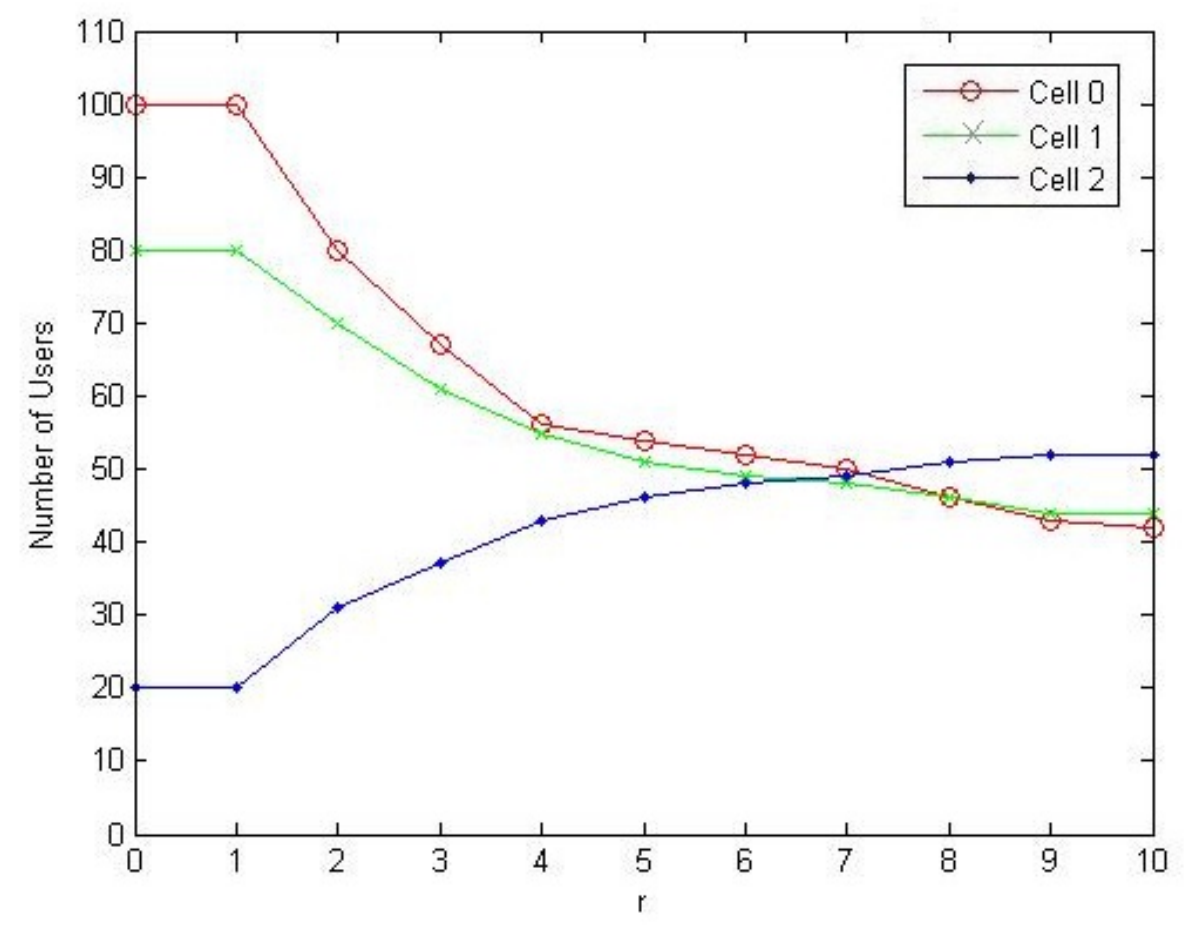

Figure 6.16: Resource Fair vs $r$ for a large city

both cells 0 and 1 showing a well balanced user distribution under these channel conditions. This is again due to the effectiveness of our design parameter $r$ that targets the boundary users that the schemes are able to balance user distribution well.

Figure 6.17 shows the varinace of load in the system. Due to the severity of noisy conditions in the large city model, when our load balancing algorithm comes into effect at $r=1$, we again see a sharp decline in the load variance initially from $r=2$ till $r=4$ because of the user handoffs. Then, the variance stabilizes despite the noisy channel conditions and mildly climbs from $r=9$ to $r=10$.

We now evaluate our schemes in terms of the load balance index of the whole system [42] now for the large city model. It can be observed from 


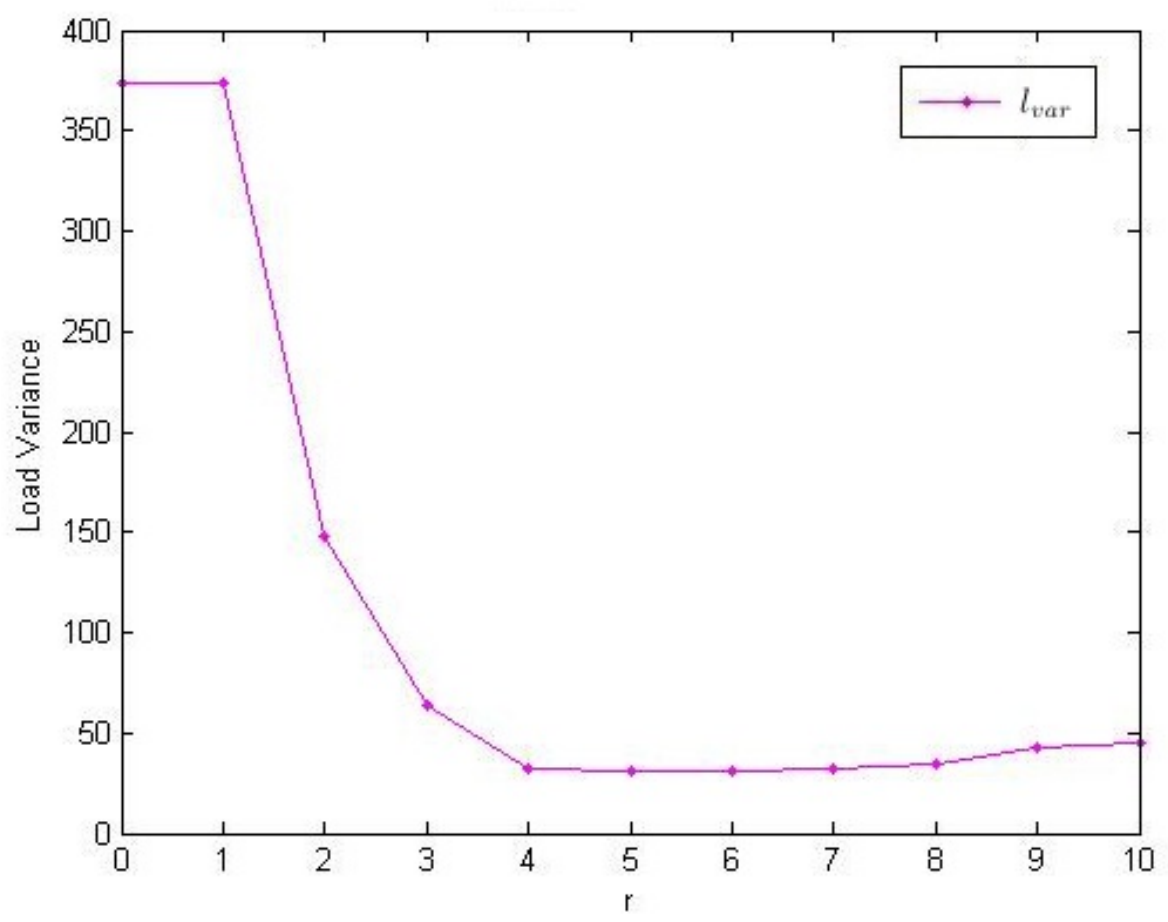

Figure 6.17: Load Variance vs $r$ for a large city

Figure 6.17 that due to the extremely noisy channel conditions, at $r=2$, when our load balancing scheme comes into effect, we see an increment initially, but it is met by strong resistance due to the noise from $r=3$ to 8 , until it sharply increases again at $r=9$. This shows the resilience of our proposed schemes in very noisy environments.

In the next section, we move on to comparing our schemes with the conventional RSSI-based association to evaluate performance comparison. 


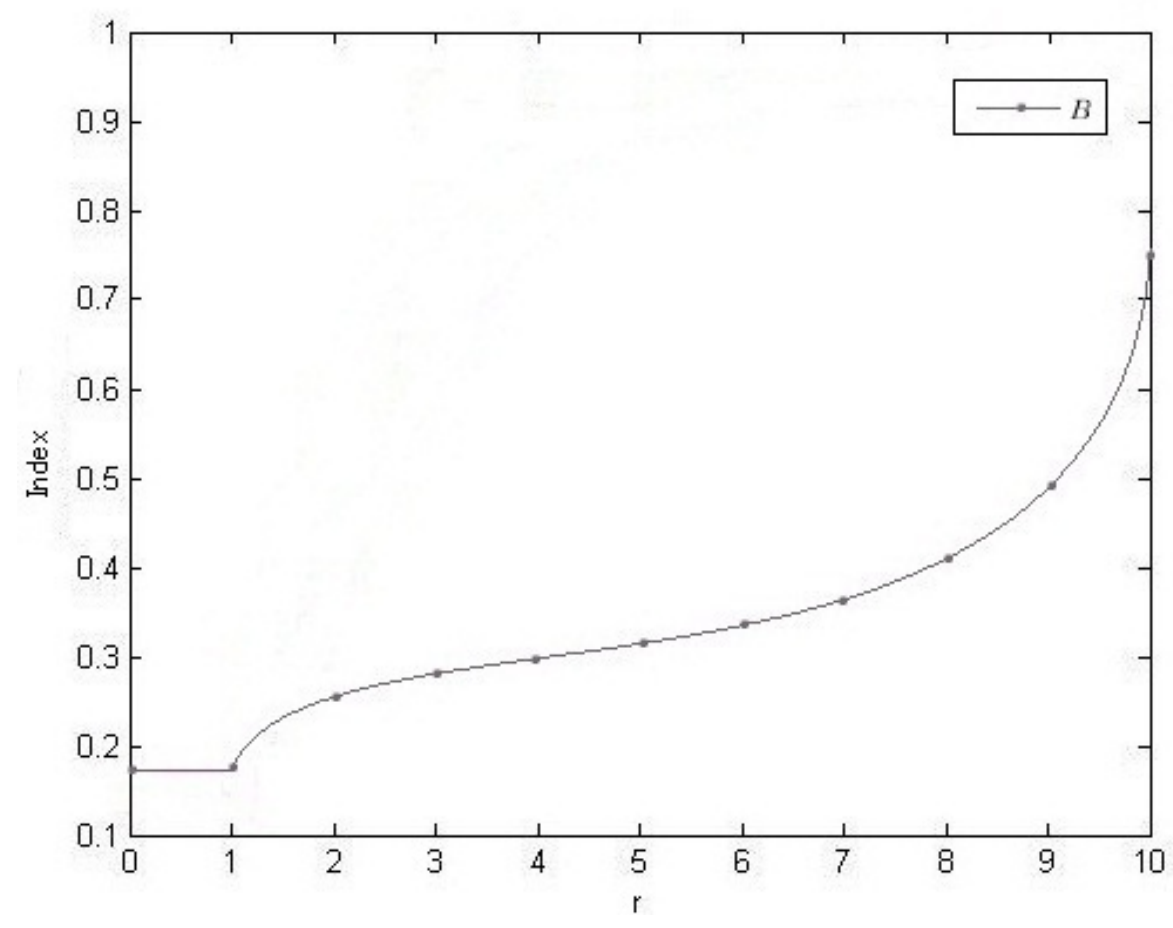

Figure 6.18: Load Balance Index vs $r$ for a large city

\subsection{Comparsion against RSSI-based Schemes}

We peform a comparison of our proposed schemes with schemes and literature based on conventional RSSI-based cell selection [47]-[48] to evaluate the performance. We use our design parameter $r$ to perform the comparison again. Figure 6.19 shows the result and demonstrates that employing our proposed association control based schemes result in a substantial improvement in the throughput of users belonging to cells 0 to 2 as compared to the conventional RSSI-based user association in each cell.

Thus, our schemes perform well when throughput fair scheduler is adopted in each cell. Finally, we perform a comparison using Jain's fairness index in Figure 6.20 and it is clearly evident that our schemes outperform the conventional RSSI based schemes in terms of an overall fairness 


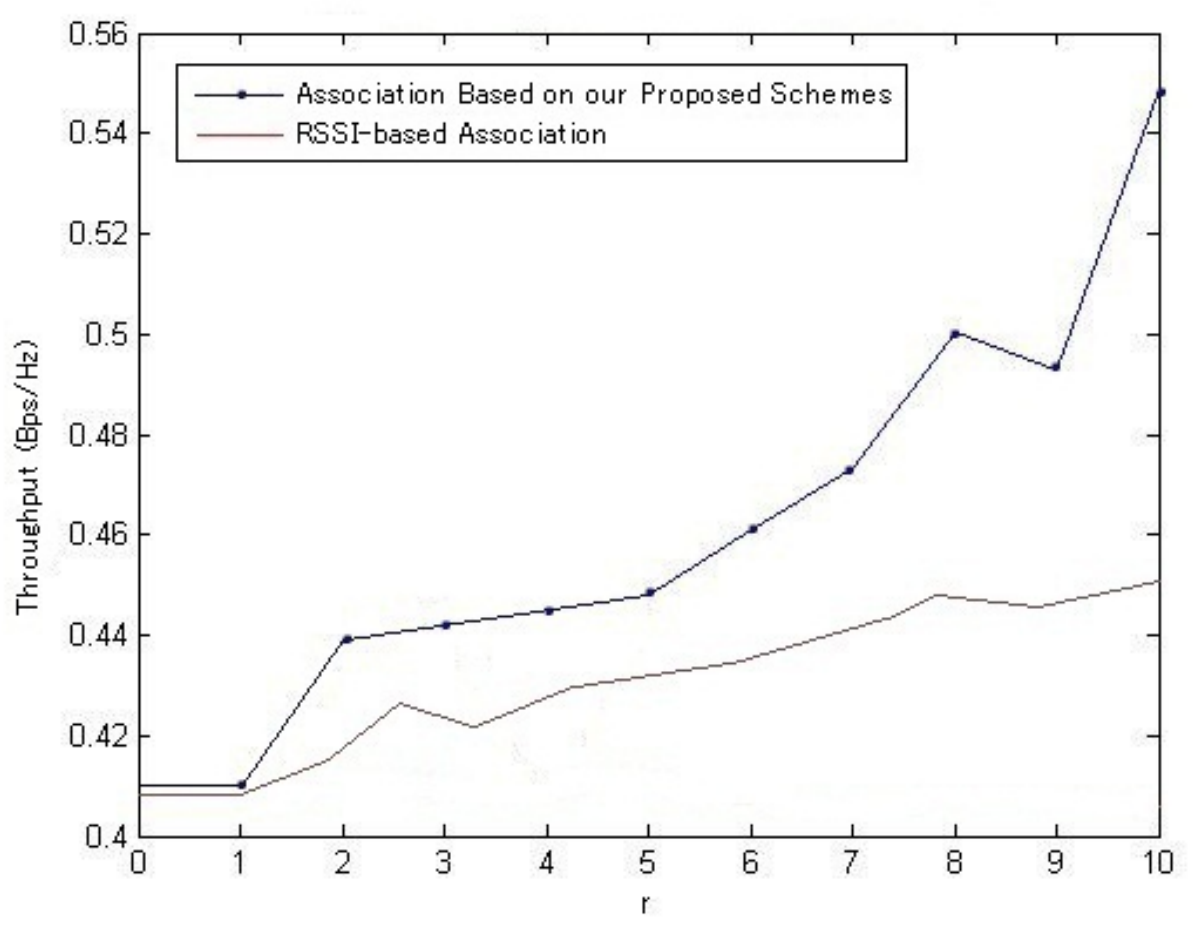

Figure 6.19: Minimum Throughput gain vs $r$

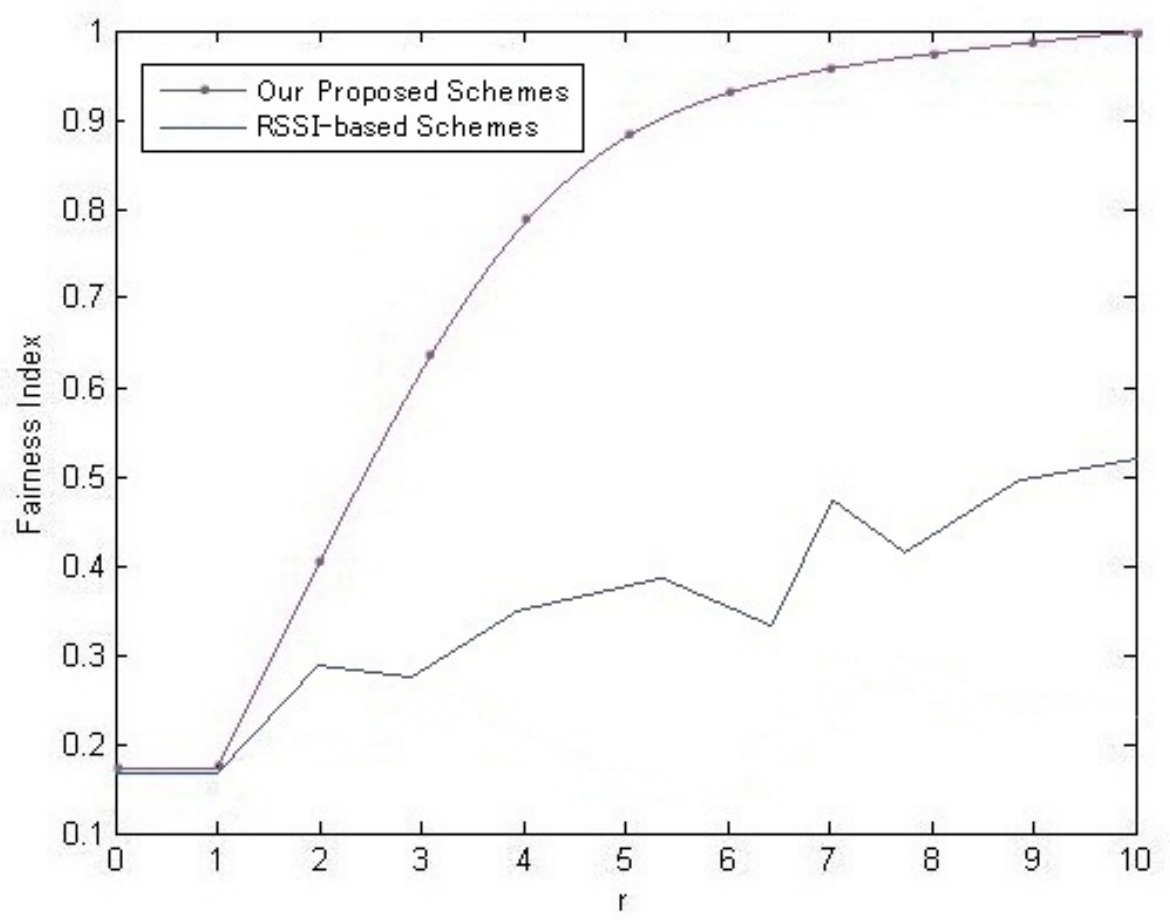

Figure 6.20: Jain's Fairness Index vs $r$ 
to users index.

\subsection{Summary}

In this chapter, we performed extensive simulations on our two proposed load balancing schemes. We first present a brief discussion on the channel characteristics of wireless networks and the various degradation factors. Then we discuss the simulation environment with the underlying assumptions. We first simulate using the peformance metric, load variance, which shows that our proposed schemes peform well balancing the load of the system, minimizing the traffic load variance. Then, the schemes are tested for fairness to users in terms of resources under various user densities to demonstrate their effectiveness in balanced user distribution across the network. We then quantify the fairness acheived by the schemes using Jain's fairness index, which displays very high levels of fairness in terms in terms of load and user distribution. We also compare the performance of our schemes under different environments to test their robustness using the Hata model. Finally, we compare our proposed schemes with schemes based on RSSI-based assocation to compare their performance and make our case. 


\section{Chapter 7}

\section{Conclusion}

Wireless communications are gaining momentum in mobile applications and the increasing demand for all types of wireless services (voice, data, multimedia) is fueling the need for higher capacity. The conventional cellular networks are experiencing several difficulties for reaching this apex and are bounded by factors such as bandwidth, coverage area, or infrastructure costs. This thesis is devoted to the development of algorithms that explore and make use of preamble sequences to alleviate the problem of non-uniform user distribution in cellular networks due to the conventional RSSI-based association. This problem is one of major degradation factors in wireless cellular networks impacting capacity and performance due to the imbalanced load across the network.

We have developed two schemes to tackle this problem in this thesis. We first developed a design parameter to work with our schemes that targets the cell-edge users for load balancing as they are the most important entities of any load balancing scheme. In our first scheme, which is the auto-correlation based implicit load indication scheme, we modify the preamble code sequence and propose the auto-correlation operation as a way to implicitly decipher the load at the BSs. This scheme allows the mobile users to make an intelligent decision while choosing a BS for service, by not only considering the strength of the received signal from the BS, 
but also the level of congestion at it. The second scheme works using the cross-correlation property among preamble sequences to implicitly obtain the load state of BSs for load balancing. This enables the users while joining the network to perform smart cell-site selection based on the level of congestion at the BS along with the conventional association metrics.

We have performed extensive tests to evaluate the performance of the schemes considering the scheduling disciplines RF and TF, as the resource allocation policies. The proposed schemes are able to obtain a high degree of improvement in the minimum throughput of users in the cells we focus on, and apply our load balancing schemes to, and perform well in terms of throughput fair scheduling. The schemes are then tested for fair user distribution and achieve great results in alleviating the problem of load variance by swiftly inducing user handoffs into lightly loaded cells from the highly loaded ones to balance the load. We also test the schemes in terms of Jain's fairness index for resource fairness evaluation and for load variance and obtain great results. Finally, we tested the schemes for robustness and compared them with schemes relying on conventional RSSI-based cell selection to the conclude that, our proposed schemes are reasonably robust, performorming well in noisy environments and outpeforming the schemes relying on the conventional association metric.

To conclude, we have confirmed the applicability of the preamble sequence as an implicit information indicating tool and stations can take advantage of this in selecting a more appropriate BS, thereby maintaining resource and throughput fairness across the network. 


\section{Chapter 8}

\section{Future Work}

Probing deeper, the results in this thesis provide a strong foundation for future work in the area of using of preamble sequences for load balancing in cellular networks. Some of the areas that can be considered for for future work are as follows:

- Developing new preamble sequence structures that will be able to accomodate and minimize degradation of the preamble sequence in terms of detection when we try to concatenate repeated PN sequences to form premables, based on the proposed auto-correlation scheme.

- There is a significant amount of computation involved in the crosscorrelation operation requiring much computation power. Therefore, developing methods which are less computationally complex will be a great benefit as they will improve the speed of cell-selection decision making and handovers.

- Lastly, it will be very interesting to see how the schemes perform in different topologies with a change in the scalability of the system, which will give a better insight into the behaviour in new dimensions. 


\section{Bibliography}

[1] V . H. Mac Donald, "The Cellular Concept," Bell Sys. Tech. J, vol. 58, no.1, pp. 15.45, Jan. 1979.

[2] T. Rapport,"Wireless Communications: Principles and Practice," Prentice Hall, 1996.

[3] A. S. Tanenbaum, Computer Networks, 4th edition, Prentice Hall, 2003.

[4] Ankit Chopra, Peter Sam Raj and Winston KG Seah, "Utilizing the Inherent Properties of Preamble Sequences for Load Balancing in Cellular Networks", accepted by the 7th International Conference on Intelligent Sensors, Sensor Networks and Information Processing (ISSNIP 2011), Adelaide, Australia, Dec 6-9, 2011.

[5] I Katzela and M. Naghshineh, "Channel Assignment Schemes for Cellular Mobile Telecommunication Systems: A Comprehensive Survey," IEEE Pers. Commun., vol.3, no.3, pp.10-31, Jun 1996.

[6] J. C.- I Chuang, "Performance Issues and Algorithms for Dynamic Channel Assignment," IEEE J. Sel. Areas Commun., vol.11, no.6, pp.955963, Aug 1993. 
[7] M. Zhang, T.- S. P. Yum , "Comparisons of Channel-Assignment Strategies in Cellular Mobile Telephone Systems," IEEE Trans. Vehic. Tech., vol.38, no.4, pp.211-215, Nov 1989.

[8] T. J. Kahwa and N. D. Georganas, "A Hybrid Channel Assignment Scheme in Large-Scale, Cellular-Structured Mobile Communication Systems,"IEEE Trans. Commun., vol.26, no.4, pp. 432- 438, Apr 1978.

[9] S. M. Elnoubi, R. Singh, and S. C. Gupta, "A New Frequency Channel Assignment Algorithm in High Capacity Mobile Communication Systems," IEEE Trans. Vehic. Tech., vol.31, no.3, pp. 125- 131, Aug 1982.

[10] H. Jiang and S.S. Rappaport, "CBWL: A New Channel Assignment and Sharing Method for Cellular Communication Systems," IEEE Trans. Vehic. Tech., vol.43, no.2, pp.313-322, May 1994.

[11] S. K. Das, S. K. Sen, and R. Jayaram, "A Novel Load Balancing Scheme for the Tele-Traffic Hot Spot Problem in Cellular Networks."Wireless Networks, vol. 4, no. 4, pp. 325-340, Jul 1998.

[12] B. Eklundh,"Channel Utilization and Blocking Probability in a Cellular Mobile Telephone System with Directed Retry," IEEE Trans. Commun., vol.34, no.4, pp. 329- 337, Apr 1986.

[13] J. Karlsson and B. Eklundh, "A Cellular Mobile Telephone System with Load Sharing Enhancement of Directed Retry," IEEE Trans. Commun., vol.37, no.5, pp.530-535, May 1989.

[14] S. S. Rappaport and L.-R. Hu, "Microcellular Communication Systems with Hierarchical Macrocell Overlays: Traffic Performance Models and Analysis," in Proc. IEEE, vol.82, no. 9, pp. 1383-1397, Sep. 1994. 
[15] I. Papanikos and M. Logothetis, "A Study on Dynamic Load Balance for IEEE 802.11b Wireless LAN" In Proc. COMCON, Crete, Greece, pages 1-7, 14-17 June, 2001.

[16] I. Tinnirello and G. Bianchi," A Simulation Study of Load Balancing Algorithms in Cellular Packet Network" In Proc. MSWIM, pages 73-78, Rome, Italy, 16-21 July, 2001.

[17] A. Balachandran, P. Bahl, and G. M. Voelker, "Hot-spot Congestion Relief and Service Guarantees in Public-Area Wireless Networks", ACM SIGCOMM Computer Communication Review., vol. 32, no.1, page 59, January, 2002.

[18] H. Velayos, V. Aleo and G. Karlsson," Load balancing in overlapping wireless LAN cells", In Proc. ICC, Vol. 7, page 3833- 3836, 20-24 June, 2004.

[19] A. Kumar and V. Kumar, "Optimal Association of Stations and APs in an IEEE 802.11 WLAN" In Proc. MSWIM, vol.1, page 1-5, Kharagpur, India, 28-30 January, 2005.

[20] T-C. Tsai and C-F. Lien., "IEEE 802.11 Hot Spot Load Balance and QoS Maintained Seamless Roaming", In Proc. NCS, page 1-8, Taiwan, R.O.C, 24-26 November, 2003.

[21] Y. Bejerano S-J. Han and L. E. Li. "Fairness and Load Balancing in Wireless LANs Using Association Control", In Proc. MOBICOM, pages 315-329, Philadelphia, PA, USA, 26 September- 01 October, 2004.

[22] Y. D. Lin and Y. C. Hsu, "Multihop cellular: A new architecture for wireless communication", in Proc. IEEE INFOCOM, page 1273- 1282, Tel- 
Aviv, Israel, 26-20 March, 2000.

[23] X. Wu, B. Mukherjee, and S. -H. G. Chan, "MACA - An Efficient Channel Allocation Scheme in Cellular Networks," in Proc. IEEE GLOBECOM, vol. 3, pp. 1385-1389, San Francisco, California, USA, 27 November- 1 Decemeber, 2000.

[24] H. Luo, R. Ramjee, P. Sinha, L. Li, and S. Lu, "UCAN: A Unified Cellular and Ad-hoc Network Architecture," in Proc. MOBICOM, pp. 353-367, San Diego, California, USA , 14-19 September, 2003.

[25] G. N. Aggelou and R. Tafazolli, "On the Relaying Capability of NextGeneration GSM Cellular Networks,"IEEE Pers. Comm., pp. 40-47, Feb. 2001.

[26] R. Yates and C.-Y. Huang, Integrated Power Control and Base Station Assignment, IEEE Trans. on Veh. Technol., vol. 44, no. 3, pp. 638-644, Aug. 1995.

[27] S. V. Hanly, "An Algorithm for Combined Cell-Site Selection and Power Control to Maximize Cellular Spread Spectrum Capacity," IEEE J. Select. Areas in Comms., vol. 13, no. 7, pp.1332-1340, Sep 1995.

[28] Aimin Sang, et al., "Coordinated Load Balancing, Handoff/Cell-site Selection and Scheduling in Multi-Cell Packet Data Systems," in Proc. ACM MobiCom, Philadelphia, PA, USA, Sep 26-Oct 1, 2004.

[29] S. Das, et al. "Dynamic Load Balancing through Coordinated Scheduling in Packet Data Systems," in Proc. IEEE INFOCOM, San Francisco, CA, USA, Apr 1-3, 2003. 
[30] Minsoo Na, Youngkyu Choi, Dongmyoung Kim, Kwanghun Han, and Sunghyun Choi, "On Utilizing Preamble Code Sequence as Implicit Load Indicator," in Proc. IEEE APWCS, Sendai, Japan, Aug 21-22, 2008.

[31] IEEE 802.16e-2006, "Part 16: Air Interface for Fixed and Mobile Broadband Wireless Access Systems-Amendment for Physical and Medium Access Control Layers for Combined Fixed and Mobile Operation in Licensed Bands," IEEE std., Feb. 2006.

[32] Byeong Gi Lee and Sunghyun Choi, Broadband Wireless Access E Local Networks: Mobile WiMAX and WiFi, Aretech House, 2008.

[33] Peng Cheng, et al., "A study on cell search algorithms for IEEE 802.16e OFDMA systems," in Proc. IEEE WCNC, Hong Kong, Mar 11-15, 2007.

[34] Shannon, et al.,"Communication in the Presence of Noise," in Proc. IEEE, Sept ,1984.

[35] De S, Tonguz O, Wu H, Qiao C."Integrated Cellular and Ad Hoc Relay (iCAR) Systems pushing the performance limits of conventional wireless networks," In Proc. IEEE HICSS, 3899-3906, January 2002.

[36] Zhou J and Yang Y. PARCelS,"Pervasive Ad-Hoc Relaying for Cellular Systems", in Proc. Med-Hoc-Net, page 1-7, Sardegna, Italy, 4-6 September 2002.

[37] T. Togo, I. Yoshii and R. Kohno, "Dynamic Cell-Size Control According to Geographical Mobile Distribution in a DS/CDMA Cellular System", In Proc. IEEE PIMRC, Vol. 2, pages 677-681, Boston, MA, USA, 8-11 September 1998. 
[38] A. Jalali,"On Cell Breathing in CDMA Networks",In Proc. IEEE ICC, Vol. 2, pages 985 - 988, Atlanta, Georgia, USA, 1 June 1998.

[39] Jerry D. Gibson, "The Mobile Communications Handbook", chap. 8, 1999.

[40] Bracewell, R. "The Autocorrelation Function." The Fourier Transform and Its Applications. New York: McGraw-Hill, pp. 40-45, 1965.

[41] Asrar U. H. Sheikh, "Wireless communications: Theory and Techniques", chap. 10, 2004.

[42] R. Jain, D. Chiu, and W. Hawe, "A Quantitative Measure Of Fairness And Discrimination For Resource Allocation In Shared Computer Systems", DEC Research Report TR-301, September 1984.

[43] B. Radunovic and J.-Y. L. Boudec, "A Unified Framework for Max-Min and Min-Max Fairness with Applications," in Proc. Allerton, Monticello, Illinois, USA, 3 October, 2002.

[44] H. J. Kushnerand P. A. Whiting, "Convergence of Proportional-Fair Sharing Algorithms under General Conditions", IEEE Trans. Wireless Commun., vol.3, no. 4, pp. 12501259, Jul 2004.

[45] D. Julian, M. Chiang, D. O'Neill, and S. Boyd, "QoS and Fairness Constarined Convex Optimization of Resource Allocation for Wireless Cellular and Ad-hoc Networks," in Proc. of IEEE INFOCOM, vol. 2, pp. 477-486, San Francisco, California USA, 30 March, 2003.

[46] H. J. Zepernick and a. Finger, "Pseudo Random Signal Processing", John Wiley and Sons Ltd., Chichester, UK, 2005. 
[47] A. Balachandran, G.M. Voelker, P. Bahl, and P.V. Rangan, Characterizing User Behavior and Network Performance in a Public Wireless LAN, ACM SIGMETRICS 2002, June 2002.

[48] D. Kotz and K. Essien, Characterizing Usage of a Campus-wide Wireless Network, Technical Report TR2002-423, Dartmouth College, March 2002.

[49] Seybold, John S., "Introduction to RF propagation" ,John Wiley and Sons, 2005. 